06

\title{
Термооптические и диэлектрические исследования сегнетоэлектрической фазы, индуцированной кальцием, в виртуальном сегнетоэлектрике $\mathrm{SrTiO}_{3}$
}

\author{
(C) П.А. Марковин ${ }^{1}$, В.А. Трепаков ${ }^{1,2}$, М.Е. Гужва ${ }^{1,3}$, A. Dejneka ${ }^{2}$, А.Г. Раздобарин ${ }^{1}$, О.Е. Квятковский1 \\ ${ }^{1}$ Физико-технический институт им. А.Ф. Иофрфе РАН, \\ Санкт-Петербург, Россия \\ ${ }^{2}$ Institute of Physics AS CR, \\ Prague, Czech Republic \\ ${ }^{3}$ Горный университет, \\ Санкт-Петербург, Россия \\ E-mail: P.Markovin@mail.ioffe.ru
}

(Поступила в Редакцию 27 марта 2018 г.)

Исследованы температурные изменения рефракции света, двупреломления, диэлектрической проницаемости и петель диэлектрического гистерезиса в монокристаллах $\mathrm{Sr}_{1-x} \mathrm{Ca}_{x} \mathrm{TiO}_{3}$ с $x=0.014$ (SCT-1.4). Диэлектрические свойства исследованы в $\mathrm{Sr}_{1-x} \mathrm{Ca}_{x} \mathrm{TiO}_{3}$ с $x=0.007$ (SCT-0.7). Выполнены расчеты ab initio равновесных структур и полных энергий трех низкотемпературных фаз для $\mathrm{SrTiO}_{3}$ и $\mathrm{CaTiO}_{3}$, на основании которых определена ожидаемая симметрия основного состояния их твердого раствора и направления спонтанной поляризации в индуцированной кальцием сегнетофазе в $\mathrm{Sr}_{1-x} \mathrm{Ca}_{x} \mathrm{TiO}_{3}$. В SCT-1.4 выделен спонтанный вклад в рефракцию света, возникающий за счет спонтанного электрооптического эффекта, вызванного спонтанной поляризацией и ее флуктуациями. Из спонтанного вклада в рефракцию света на основе ранее развитого авторами фенологического подхода впервые количественно определены величины и температурные зависимости среднеквадратичных флуктуаций параметра порядка - поляризации $P_{\mathrm{sh}}=\left\langle P_{\mathrm{fl}}^{2}\right\rangle^{1 / 2}$ (ближний, локальный полярный порядок) в сегнетоэлектрической фазе. Из оптических и диэлектрических измерений в SCT-1.4 определено среднее значение спонтанной поляризации $P_{s}$ (вклад дальнего порядка). Проведены оценки величин $P_{\mathrm{sh}}$ и $P_{s}$, характеризующих ближний и дальний порядок в сегнетофазе SCT-0.7. Определение в отдельности величин и температурных зависимостей $P_{s}$ и $P_{\mathrm{sh}}$, которая значительно превышает величину $P_{s}$ в упорядоченной фазе, позволило на количественном уровне выявить новые особенности формирования индуцированной полярной фазы в $\mathrm{Sr}_{1-x} \mathrm{Ca}_{x} \mathrm{TiO}_{3}$.

П.А. Марковин благодарен программе президиума РАН „Физика конденсированных сред и материалы нового поколения“, В.А. Трепаков благодарен программе „Современные проблемы физики низких температур“ за частичную финансовую поддержку работы.

DOI: 10.21883/FTT.2018.09.46393.081

\section{1. Введение}

Титанат стронция $\mathrm{SrTiO}_{3}$ (STO) является виртуальным сегнетоэлектриком типа смещения [1]. Принято считать [1], что, как и в $\mathrm{KTaO}_{3}$ (КТО), нулевые колебания решетки стабилизируют параэлектрическое состояние (ПС) в STO в области низких температур (квантовое параэлектричество). В отличие от КТО, где кубическая фаза сохраняется вплоть до $0 \mathrm{~K}$, в STO при $T_{0}=105 \mathrm{~K}$ происходит структурный фазовый переход в антиферродисторсионную (АФД) фазу с тетрагональной симметрией. В работе [2] показано, что искажение решетки в АФД фазе также подавляет сегнетоэлектрическую (СЭ) неустойчивость решетки. При этом оказывается [3], что лишь совместное действие квантовых эффектов и тетрагонального искажения решетки стабилизирует ПС в STO, так как кубическая фаза была бы неустойчивой даже с учетом влияния нулевых колебаний.

Допирование небольшим количеством примесей (или изотопное замещение ${ }^{16} \mathrm{O}$ на ${ }^{18} \mathrm{O}$ в $\left.\mathrm{SrTiO}_{3}\right)$ с концентрацией выше некоторой критической $x_{\mathrm{c}}$ приводит к се- гнетоэлектрическому фазовому переходу $[1,4-10]$. Многочисленные исследования таких систем $\left(\mathrm{Sr}_{1-x} \mathrm{Ca}_{x} \mathrm{TiO}_{3}\right.$, $\mathrm{Sr}_{1-x} \mathrm{Ba}_{x} \mathrm{TiO}_{3}, \mathrm{KTa}_{1-x} \mathrm{Nb}_{x} \mathrm{O}_{3}, \operatorname{SrTi}\left({ }^{16} \mathrm{O}_{1-x}{ }^{18} \mathrm{O}_{x}\right)^{3}$ и др. $)$ указывают на сосуществование ближнего и дальнего полярного порядка в индуцированной примесями полярной фазе [4-8,11-22]. Однако до настоящего времени экспериментальные данные позволяли сделать только качественное описание сосуществования ближнего и дальнего порядка в сегнетофазе. Наглядным примером являются работы по рефрактометрическим исследованиям таких систем [14-22]. Измерения температурных изменений главных значений показателя преломления $\delta n_{i}(T)$ после выделения спонтанного полярного вклада $\delta n_{i}^{s}(T)$ в показатель преломления, обусловленного квадратичным по поляризации электрооптическим (ЭО) эффектом [23,24], позволяют определить среднее (по времени и объему кристалла) значение квадрата спонтанной поляризации $\delta n^{s}(T) \propto\left\langle P^{2}\right\rangle$, которое включает в себя вклад ближнего и дальнего полярного порядка $[17,18,25,26]$. Подобную информацию в некубических кристаллах можно получить и из спонтанного полярного вклада 
$\Delta n^{s}(T) \propto\left\langle P^{2}\right\rangle$ в температурные зависимости кристаллографического двупреломления $\Delta n(T)[14-16,19,21,22]$. В индуцированной примесями сегнетофазе в $\mathrm{SrTiO}_{3}$ и $\mathrm{KTaO}_{3}$ величина $\left\langle P^{2}\right\rangle^{1 / 2}$, включающая и дальний, и ближний порядок, оказывается значительно больше среднего значения спонтанной поляризации $P_{s}=\langle P\rangle$, что указывает на развитые пространственные флуктуации параметра порядка, характер которых качественно отличается от поведения классических термодинамических флуктуаций вблизи температуры Кюри $T_{c}$. Общей проблемой для рефрактометрических исследований систем, характеризуемых параметром порядка $\eta$, в которых наблюдается сосуществование ближнего и дальнего порядка, являлось отсутствие метода вычисления из эксперимента величины $\eta_{\mathrm{sh}}=\left\langle\eta_{\mathrm{fl}}^{2}\right\rangle^{1 / 2}$, обусловленной только ближним порядком (локальным порядком, флуктуациями параметра порядка $\left.\eta_{\mathrm{fl}}\right)$ при температурах ниже $T_{c}$. В работе [25] нами был разработан метод количественного вычисления величин $P_{\mathrm{sh}}=\left\langle P_{\mathrm{fl}}^{2}\right\rangle^{1 / 2}$ и $P_{s}=\langle P\rangle$ из спонтанного полярного вклада $\delta n^{S}(T)$ в температурные изменения главных значений показателя преломления сегнетоэлектриков на основе модификации выражений для изменений показателя преломления под действием спонтанной поляризации с учетом ее флуктуаций. Метод адаптирован для нахождения $P_{s}$ и $P_{\mathrm{sh}}$ из интерферометрических измерений. Показана возможность вычисления $P_{\mathrm{sh}}$ и $P_{s}$ непосредственно из получаемых при интерферометрических измерениях температурных изменений относительного оптического пути света в кристалле, если тем же интерферометрическим методом измерены электрооптические коэффициенты.

В настоящей работе этот метод применен для экспериментального определения величины и температурной зависимости $P_{\mathrm{sh}}=\left\langle P_{\mathrm{fl}}^{2}\right\rangle^{1 / 2}$ в сегнетоэлектрической фазе в монокристаллах $\mathrm{Sr}_{1-x} \mathrm{Ca}_{x} \mathrm{TiO}_{3}$ (SCT), в системе, в которой в сегнетофазе сосуществуют ближний и дальний полярный порядок при концентрации кальция выше критической $x_{c}=0.0018$ [10]. Вычислены величины и температурные зависимости $P_{\text {sh }}$ и $P_{s} \quad$ в $\mathrm{Sr}_{1-x} \mathrm{Ca}_{x} \mathrm{TiO}_{3}$ c $x=0.014$ (SCT-1.4). B $\mathrm{Sr}_{1-x} \mathrm{Ca}_{x} \mathrm{TiO}_{3}$ c $x=0.007$ (SCT-0.7) проведена оценка $P_{\mathrm{sh}}$ и $P_{s}$ на основе наших и литературных данных. Выполнены расчеты $a b$ initio равновесных структур и полных энергий трех низкотемпературных фаз для $\mathrm{SrTiO}_{3}$ и $\mathrm{CaTiO}_{3}$, на основании которых определена ожидаемая симметрия основного состояния их твердого раствора $\mathrm{Sr}_{1-x} \mathrm{Ca}_{x} \mathrm{TiO}_{3}$, что принципиально для корректного определения направления спонтанной поляризации $\mathbf{P}_{s}$ ниже $T_{c}$ при вычислении $P_{\text {sh }}$ из рефрактометрических измерений. Результаты работы позволили выявить на количественном уровне новые особенности формирования индуцированной полярной фазы в $\mathrm{Sr}_{1-x} \mathrm{Ca}_{x} \mathrm{TiO}_{3}$. Величина $P_{\text {sh }}$, характеризующая ближний порядок, существенно превосходит среднее значение спонтанной поляризации $P_{s}$ (вклад дальнего порядка) в упорядоченной фазе и гораздо слабее меняется с концентрацией кальция по сравнению с $P_{s}$. Предварительные результаты для SCT-1.4 опубликованы в работе [26].

\section{2. Методика эксперимента и образцы}

Температурные изменения рефракции света проводились на гомодинном интерферометре $[27,28]$, на длине волны света гелий-неонового лазера $0.6328 \mu \mathrm{m}$ в монокристаллах SCT-1.4 и номинально чистом $\mathrm{SrTiO}_{3}$ (STO). Метод позволяет измерять относительные изменения оптического пути света в кристалле $\delta \Psi(T)$, которые связаны с изменениями показателя преломления $\delta n(T)$ :

$$
\delta \Psi_{i}(T)=\delta n_{i}(T)+\left(n_{i}-1\right) \frac{\delta l_{j}(T)}{l_{j}},
$$

где $l_{j}$ - толщина образца вдоль направления распространения света, $\delta l_{j}$ - изменения толщины при внешнем воздействии (изменении температуры в данном случаe), $n_{i}$ - главные значения показателя преломления, индекс $i$ относится к направлению поляризации света, а индекс $j-$ к направлению распространения света в кристалле. Точность измерения $\delta \Psi(T)$ составляла $\approx 10^{-5}$. Двупреломление измерялось на поляриметре с точностью $\approx 10^{-6}$. Диэлектрические измерения проводились на образцах SCT-1.4 и SCT-0.7. Для измерения температурных зависимостей диэлектрической проницаемости и диэлектрических потерь на частотах $1 \mathrm{kHz}$ и $1 \mathrm{MHz}$ в качестве измерителей полного импеданса образца использовались автоматические цифровые мосты Е7-8 и Е7-12 соответственно. Относительная погрешность определения значения диэлектрической проницаемости составляла не более $0.2 \%$. Амплитуда измерительного поля для моста Е7-8 $(1 \mathrm{kHz})$ составляла $\approx 30 \mathrm{~V} / \mathrm{cm}$, а для моста $\mathrm{E} 7-12(1 \mathrm{MHz}) \approx 3 \mathrm{~V} / \mathrm{cm}$. Измерения петель диэлектрического гистерезиса и температурных зависимостей спонтанной поляризации проводились на автоматизированной установке, работающей по модифицированной схеме Сойера-Тауэра. Установка позволяла проводить измерения образцов с малыми величинами спонтанной и остаточной поляризаций в диапазоне частот $50 \mathrm{~Hz}-1 \mathrm{kHz}$. В оптических измерениях использовался гелиевый криостат, в котором образец крепился в вакууме на охлаждаемой головке, что позволяло проводить исследования в интервале $14-300$ К. Диэлектрические измерения проводились в парах гелия в диапазоне температур 5-300 K.

Использованные в работе монокристаллы $\mathrm{Sr}_{1-x} \mathrm{Ca}_{x} \mathrm{TiO}_{3}$ были выращены методом зеркальной зонной плавки в исследовательской лаборатории IBM, Цюрих, Рюшликон, Швейцария. Проведенный с помощью метода электронного микрозонда анализ химического состава и однородности распределения Са показал однородность использованных образцов с выявленными флуктуациями $\Delta x= \pm 0.0005$. Кристаллы $\mathrm{SrTiO}_{3}$ выращивались из раствора в расплаве. Монокристаллические образцы STO и SCT были изготовлены в виде прямоугольных полированных пластин с ребрами, параллельными псевдокубическим направлениям $[110]_{a}$, $[1 \overline{1} 0]_{b}$ и $[001]_{c}$ с наибольшим линейным размером 


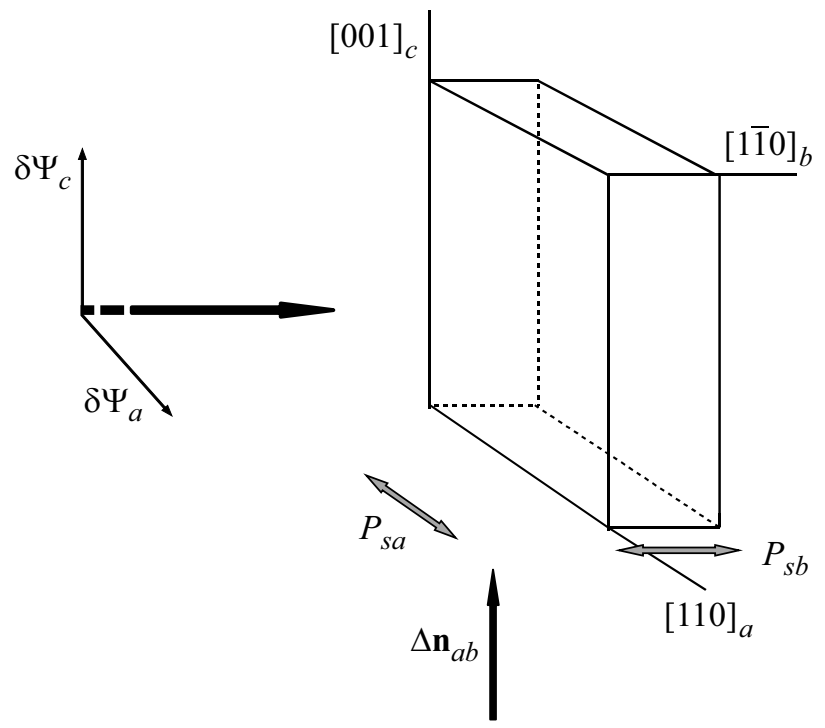

Рис. 1. Направления распространения и поляризация света по отношению к осям образца SCT-1.4 (SCT с $x=0.014)$ при измерениях изменений рефракции света $\delta \Psi_{a}, \delta \Psi_{c}$ и морфического двупреломления $\Delta n_{a b}$. Жирные черные стрелки - направления распространения света. Серые обоюдоострые стрелки возможные направления спонтанной поляризации в образце. Тонкие стрелки с обозначениями $\delta \Psi_{a}, \delta \Psi_{c}$ соответствуют направлениям поляризации света при измерениях $\delta \Psi_{a}, \delta \Psi_{c}$.

$(3.5-2.7 \mathrm{~mm})$ вдоль оси $[001]_{c}$ и $\quad$ с $\quad$ наименьшим размером $0.8 \mathrm{~mm}$ вдоль оси $[1 \overline{1} 0]_{b}$. Эти направления и обозначения $a, b, c$ относятся к тетрагональным осям ниже температуры $T_{0}$ антиферродисторсионного фазового перехода $(Ф П) \mathrm{O}_{h}-\mathrm{D}_{4 h}$ в STO и SCT. Выбранная ориентация и размеры кристаллов в соответствии с [29] позволяли надеяться на формирование структурно монодоменного образца ниже $T_{0}$. По литературным данным температуры структурного ФП $T_{0}$ в SCT-1.4 и SCT-0.7 равны соответственно $148 \mathrm{~K}$ и $125 \mathrm{~K}$, а температуры индуцированного сегнетоэлектрического перехода $T_{c}-28 \mathrm{~K}$ и $18 \mathrm{~K}[14,20,30]$. В наших измерениях температура Кюри определялась из положения максимумов диэлектрической проницаемости на частоте $1 \mathrm{kHz}$. При интерферометрических измерениях STO и SCT-1.4 свет распространялся вдоль направления $[1 \overline{1} 0]_{b}$, а поляризация света была направлена по осям $[110]_{a}$, $[001]_{c}$. Морфическое двупреломление $\Delta n_{a b}=n_{b}-n_{a}$ измерялось при направлении распространения света вдоль оси $[001]_{c}$. Направления распространения и поляризация света при оптических измерениях по отношению к осям образца изображены на рис. 1. Диэлектрические измерения проводились при приложении электрического поля вдоль $[1 \overline{1} 0]_{b}$. В качестве электродов использовалась серебряная паста. Температура образца, помещенного в гелиевый криостат, контролировалась независимо тремя датчиками - термопарами медь-константан, медь-медь с примесью железа и полупроводниковым датчиком типа КГГ и определялась с точностью $\pm 0.1 \mathrm{~K}$.

\section{3. Экспериментальные результаты}

\section{1. Диэлектрические исследования}

На рис. $2, a, b$ представлены температурные зависимости диэлектрической проницаемости в исследованных кристаллах SCT. B SCT-1.4 температурная зависимость диэлектрической проницаемости проходит через резкий максимум при температуре $T_{c}=28 \mathrm{~K}$ на частоте $1 \mathrm{kHz}$ и $29 \mathrm{~K}$ на частоте $1 \mathrm{MHz}$. При температурах, значительно больших $T_{c}$, зависимость диэлектрической проницаемости следует закону Кюри-Вейса (см. вставку на рис. $2, a)$. Определенная из этой зависимости величина постоянной Кюри имела типичную для сегнетоэлектриков типа смещения величину $C=7.7 \cdot 10^{4} \mathrm{~K}$. Характерное для систем виртуальных сегнетоэлектриков с примесями отклонение от закона Кюри-Вейса [30] при приближении к температуре перехода в индуцированную сегнетофазу обусловлено тем, что зависимость $\varepsilon(T)$ при низких температурах определяется доминирующей квантовой статистикой систем, находящихся вблизи
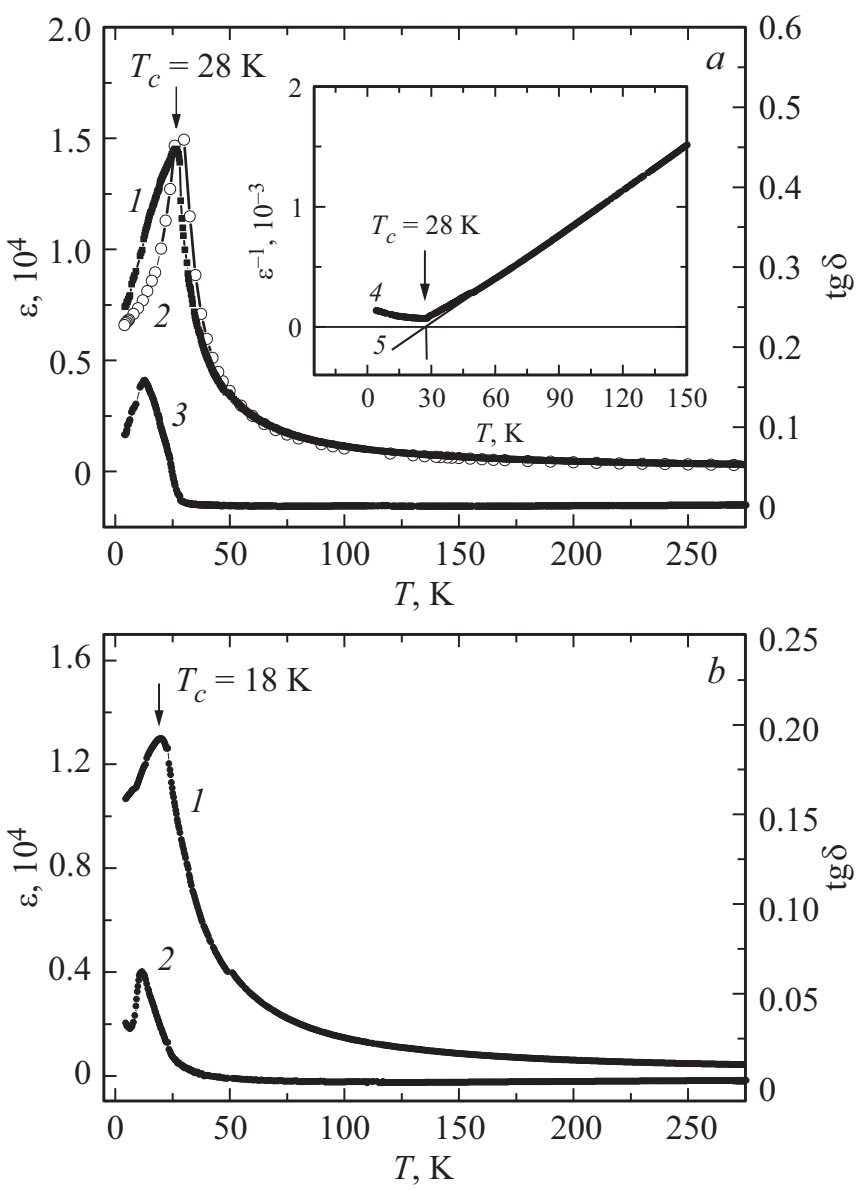

Рис. 2. $a-$ зависимости $\varepsilon(T)$ на частотах $1 \mathrm{kHz}(1)$, $1 \mathrm{MHz}(2)$ и $\operatorname{tg} \delta(T)(3)$ на частоте $1 \mathrm{kHz}$ в SCT с $x=0.014$. На вставке: зависимость $-\varepsilon^{-1}(T)(4)$ на частоте $1 \mathrm{kHz}$ и аппроксимация зависимости $\varepsilon^{-1}(T)$ функцией $A\left(T-T_{c}\right)(5)$. $b$ - зависимости $\varepsilon(T)(1)$ и $\operatorname{tg} \delta(T)(2)$ на частоте $1 \mathrm{kHz}$ в SCT c $x=0.007$. 

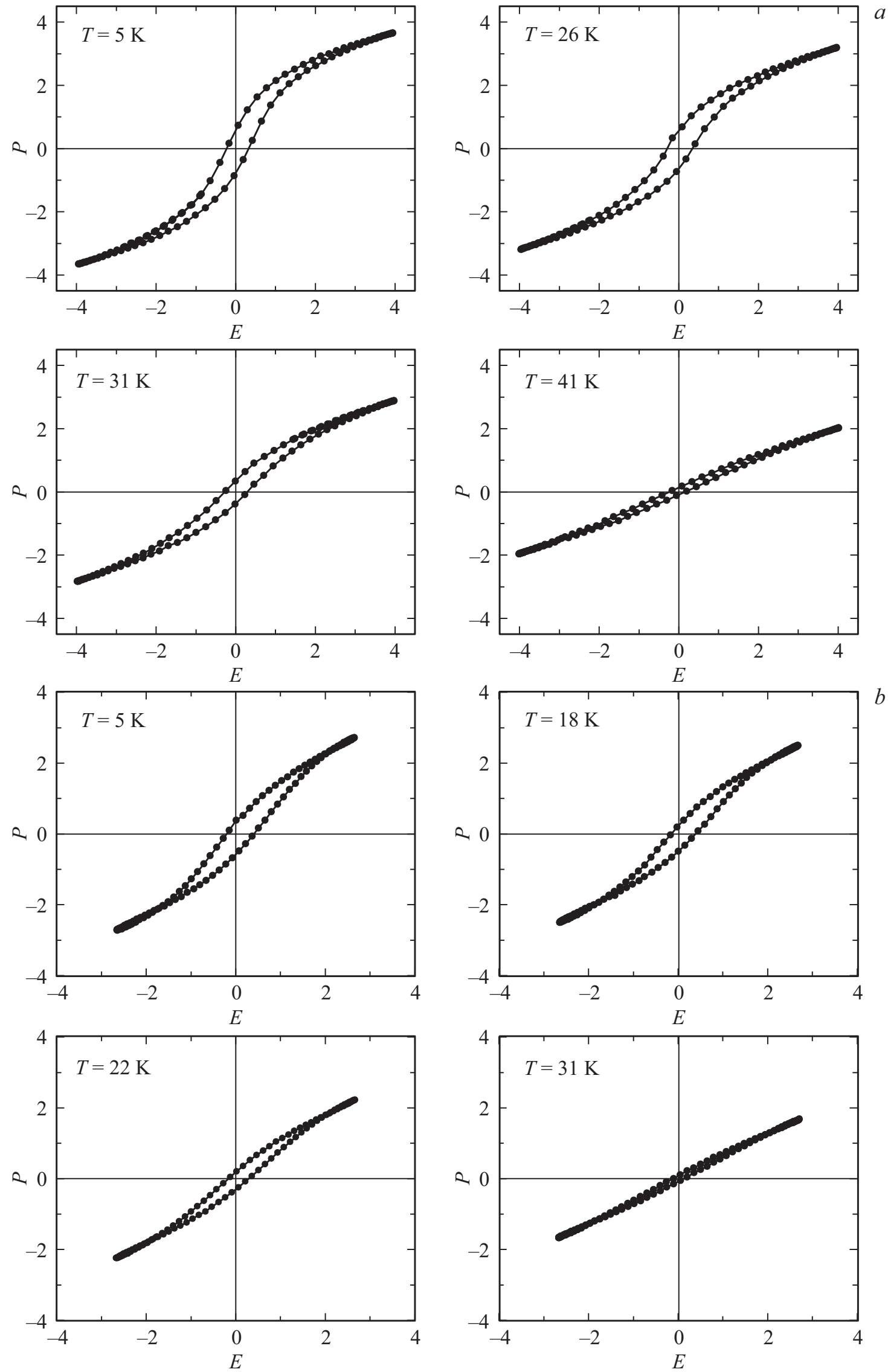

Рис. 3. $a-$ петли диэлектрического гистерезиса в SCT с $x=0.014$. Масштаб по оси $P-10^{-2} \mathrm{C} / \mathrm{m}^{2}$, по оси $E-\mathrm{kV} / \mathrm{cm}$. $b-$ петли диэлектрического гистерезиса в SCT с $x=0.007$. Масштаб по оси $P-10^{-2} \mathrm{C} / \mathrm{m}^{2}$, по оси $E-\mathrm{kV} / \mathrm{cm}$. 
квантово-механического предела [10,30,31]. Полученное значение $T_{c}=28 \mathrm{~K}$ для $x=0.014$ соответствует зависимости $T_{c}(x)$ в SCT, приведенной в [10]. В образце SCT-0.7 максимум $\varepsilon(T)$ на частоте $1 \mathrm{kHz}$ наблюдался при температуре $18 \mathrm{~K}$, что согласуется с данными, полученными для кристаллов $\mathrm{Sr}_{1-x} \mathrm{Ca}_{x} \mathrm{TiO}_{3}$ с номинально той же концентрацией кальция в $[14,20,30]$ и которую мы идентифицировали как температуру Кюри $T_{c}=18 \mathrm{~K}$. Изменение с частотой положения максимума $\varepsilon(T)$, как и в образце SCT-1.4, было незначительным, что соответствует результатам работы [30].

Для определения величины и температурной зависимости спонтанной поляризации в SCT с $x=0.014$ и 0.007 были проведены измерения петель диэлектрического гистерезиса на частоте $50 \mathrm{~Hz}$, результаты которых приведены на рис. $3, a$ и $b$ соответственно. Вид петель гистерезиса отличается от классического. Тем не менее, при увеличении электрического поля зависимость $P(E)$ выходит на линейный участок с насыщением (рис. 4, $a$ и $b)$, экстраполяция которого к $E=0$ позволяет оценить
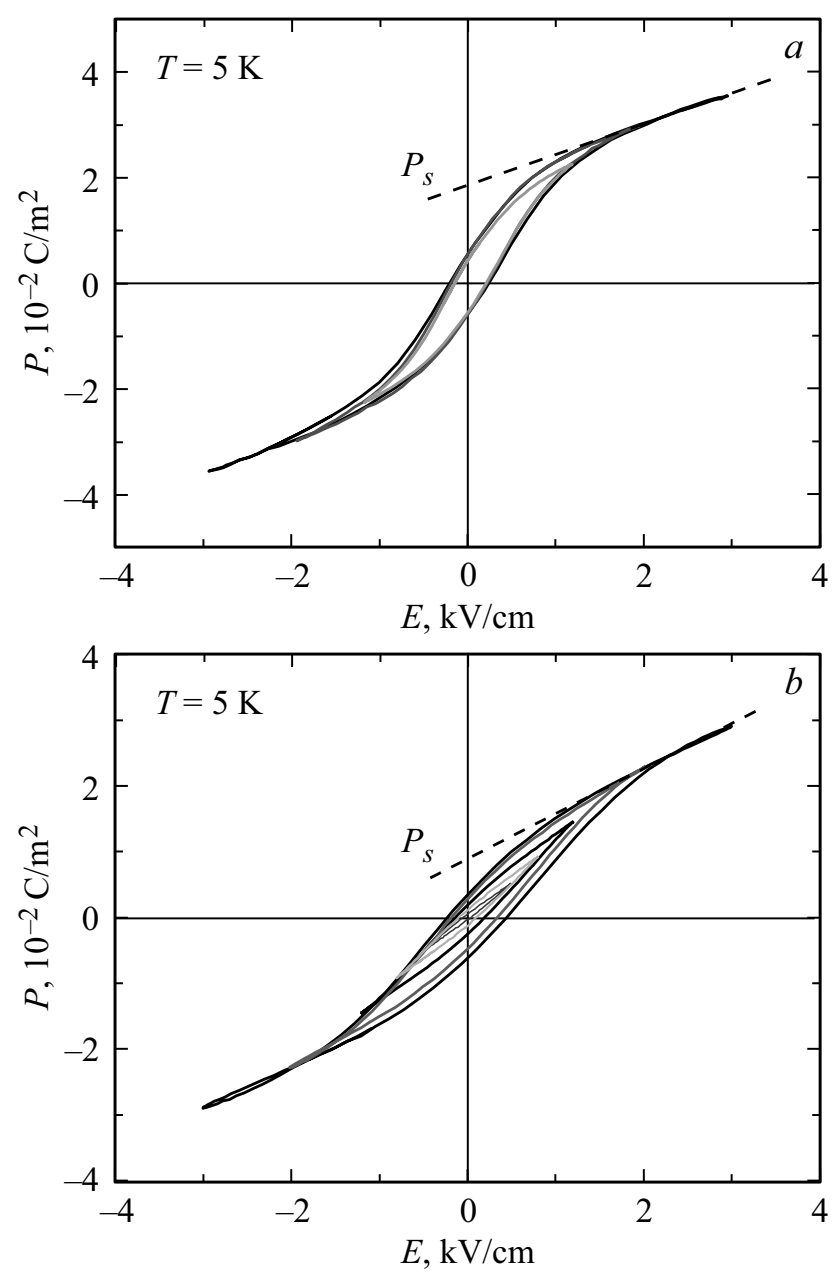

Pис. 4. $a$ - петли гистерезиса в SCT с $x=0.014$ при различных амплитудах электрического поля $E . b-$ петли гистерезиса в SCT с $x=0.007$ при различных амплитудах электрического поля $E$.

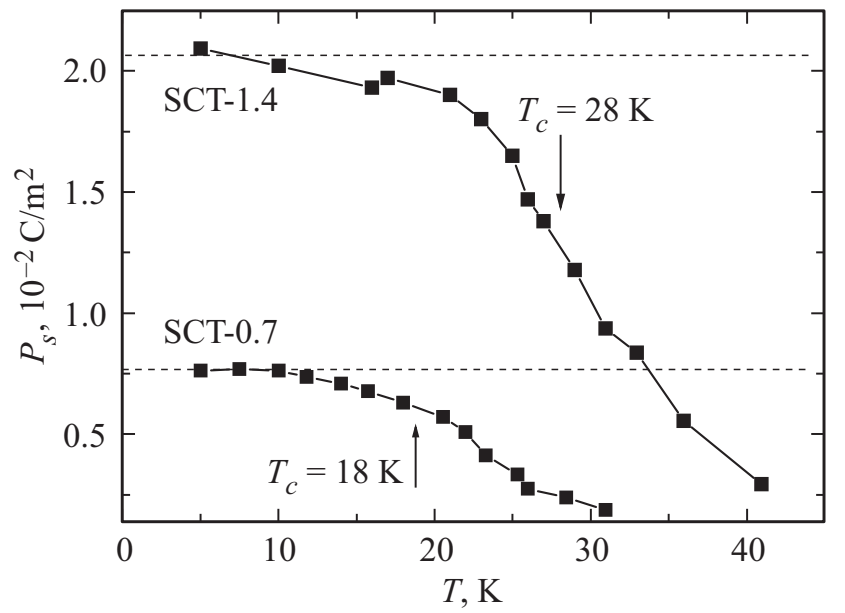

Рис. 5. Температурные зависимости среднего значения спонтанной поляризации $\left\langle P_{s}\right\rangle(T)$ в SCT-1.4 и SCT-0.7, полученные из петель диэлектрического гистерезиса.

среднее значение спонтанной поляризации $\left\langle P_{s}\right\rangle$. При температуре $T=5 \mathrm{~K}\left\langle P_{s}\right\rangle=2.1 \cdot 10^{-2} \mathrm{C} / \mathrm{m}^{2}$ в SCT-1.4 и $\left\langle P_{s}\right\rangle=7.7 \cdot 10^{-3} \mathrm{C} / \mathrm{m}^{2}$ в SCT-0.7. На рис. 5 представлена температурная зависимость среднего значения переключаемой поляризации $\left\langle P_{s}\right\rangle$ в SCT-1.4 и SCT-0.7, полученная из петель диэлектрического гистерезиса. Поскольку измерения $P(E)$ проводятся в достаточно сильных электрических полях в материале с сильной поляризуемостью решетки, то нет ничего удивительного в ,затягивании“ электрическим полем поляризации, получаемой из петель, в область температур выше $T_{c}$. Истинное температурное поведение спонтанной поляризации $\left\langle P_{s}\right\rangle$ в сегнетофазе, индуцированной примесями в виртуальных сегнетоэлектриках, можно получить из измерения спонтанных эффектов (без приложения электрического поля), например, из морфического двупреломления.

\section{2. Оптические исследования}

На рис. 6 представлены температурные изменения относительного оптического пути света $\delta \Psi_{a}$ и $\delta \Psi_{c}$ в SCT-1.4 и STO, соответствующие изменениям главных значений показателя преломления $\delta n_{a}$ и $\delta n_{c}$ (формула (1)). Индексы $a$ и $c$ соответствуют обозначениям осей образца, указанным в разделе 2 и на рис. 1 . В дальнейшем мы будем для обеих величин $\delta \Psi$ и $\delta n$ использовать термин изменения рефракции света. Температуры $T_{c}$ и $T_{0}$, обозначенные на рис. 6 стрелками, определялись как температуры максимумов производной $d\left\{\delta \Psi_{i}(T)\right\} / d T$, представленной на рис. 7. Определенная таким образом температура $28 \mathrm{~K}$ совпадает с $T_{c}$, полученной из максимума диэлектрической проницаемости. Температуры структурного фазового перехода $T_{0}$ определялись как изломы на кривых $\delta \Psi_{a}$ и $\delta \Psi_{c}$, а из производной $d\left\{\delta \Psi_{i}(T)\right\} / d T$ определялись так, как показано на рис. 7. 
Аномалии при $T_{0}$ на температурных зависимостях $\delta \Psi_{a}$ и $\delta \Psi_{c}$ выражены слабее, чем при $T_{c}$.

При извлечении количественной информации из экспериментальных зависимостей $\delta \Psi(T)$ важно корректное разделение спонтанного вклада $\delta \Psi^{s}(T)$, связанного со

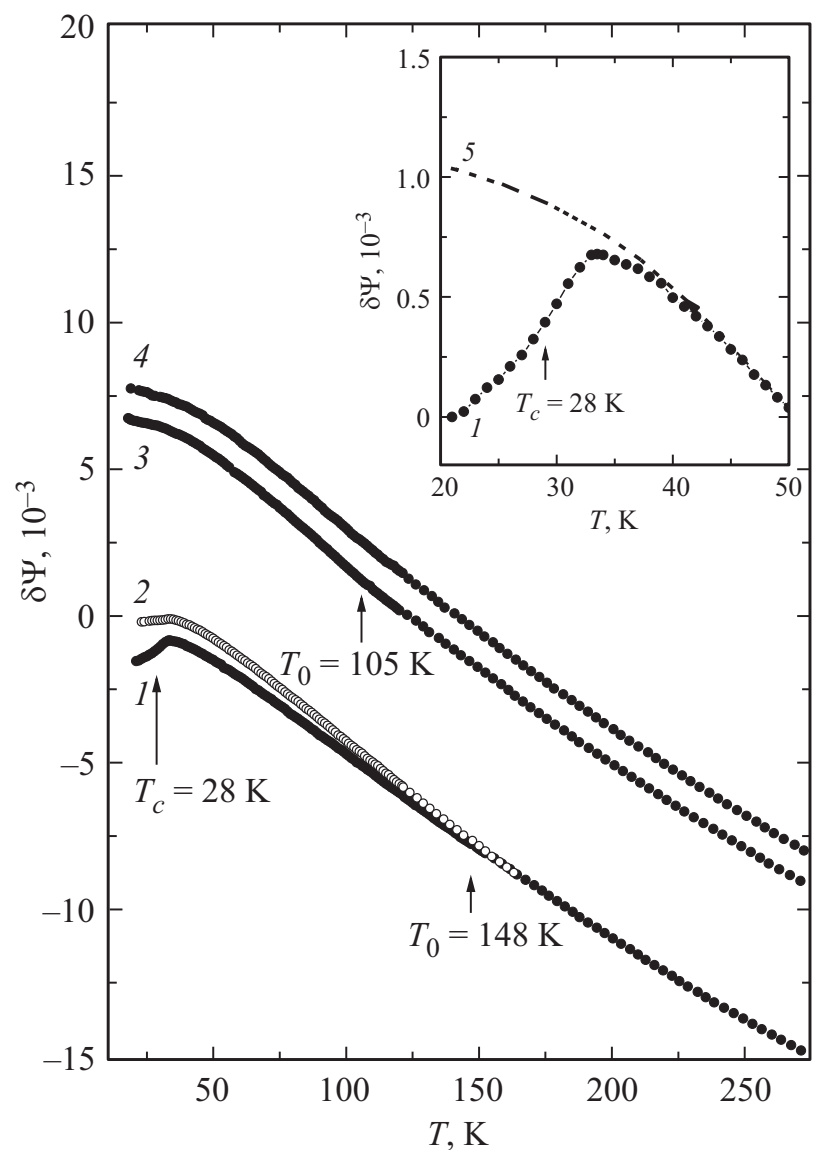

Рис. 6. Температурные зависимости $\delta \Psi_{a}(1), \delta \Psi_{c}(2)$ в SCT-1.4 и $\delta \Psi_{a}(3), \delta \Psi_{c}(4)$ в STO. На ставке фрагмент экспериментальной зависимости $\delta \Psi_{a}(T)(1)$ в SCT-1.4 и экстраполяция регулярного вклада $\delta \Psi_{a}^{0}(T)(5)$ в рефракцию света в SCT-1.4.

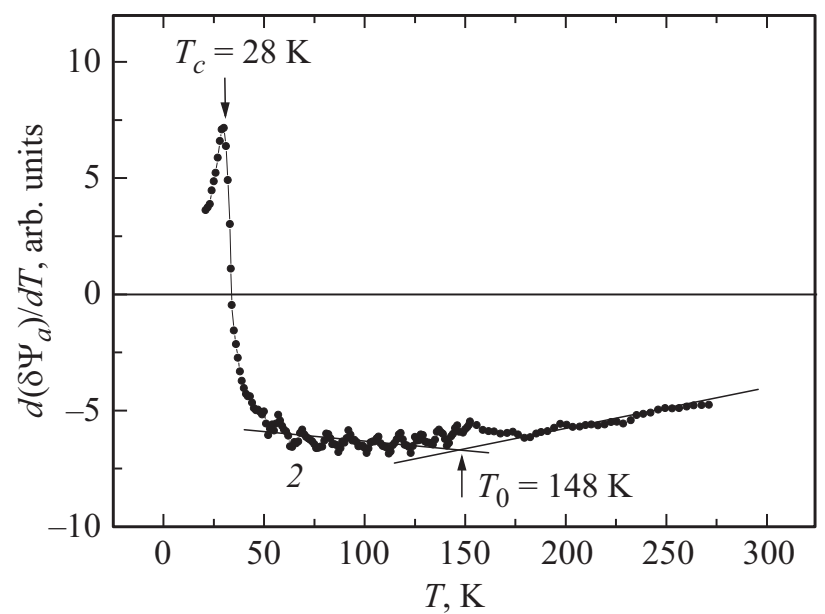

Рис. 7. Температурные зависимости производной по температуре $d\left\{\delta \Psi_{a}(T)\right\} / d T$ в SCT-1.4.

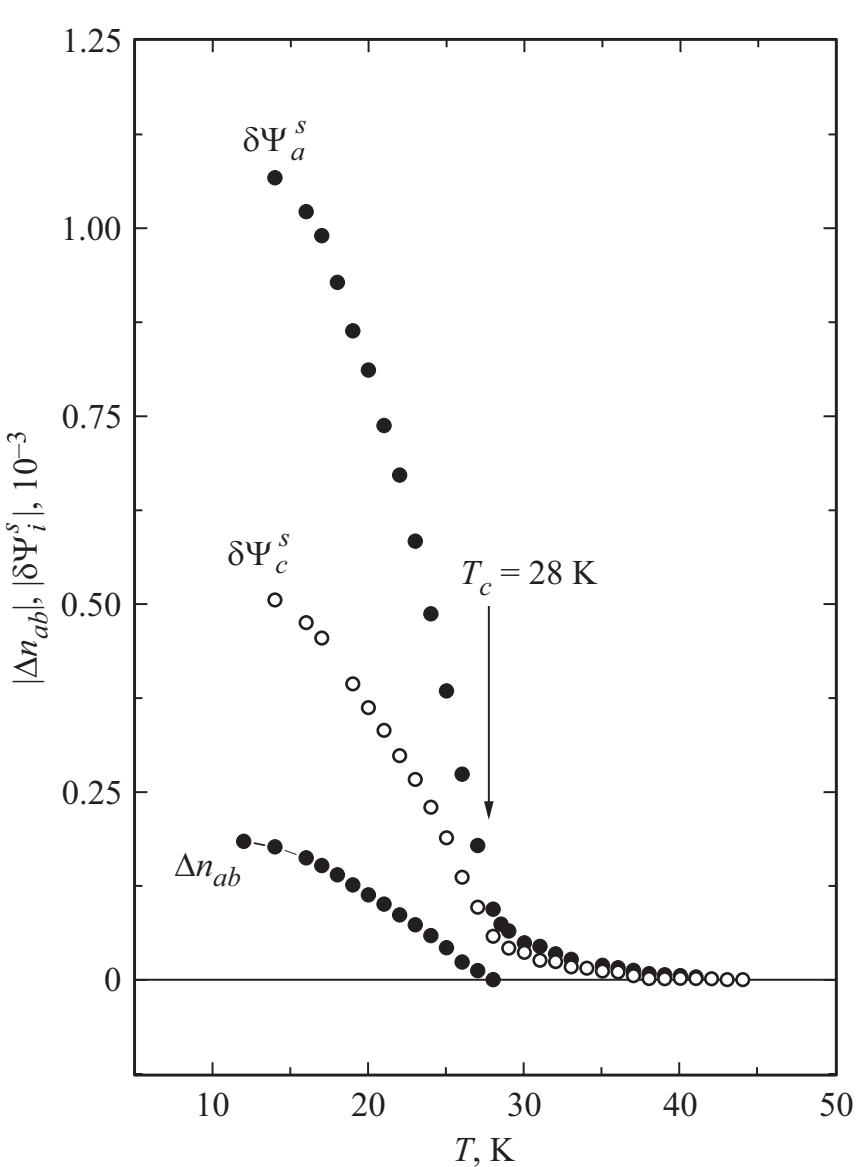

Рис. 8. Температурные зависимости спонтанного полярного вклада в рефракцию света $\delta \Psi_{a}^{S}, \delta \Psi_{c}^{S}$ и морфического двупреломления $\Delta n_{a b}$ в SCT-1.4.

спонтанной поляризацией и так называемого регулярного вклада $\delta \Psi^{0}(T)$, от нее не зависящего. Метод экстраполяции регулярного $\delta \Psi^{0}(T)$ вклада из высокотемпературной парафазы в упорядоченную фазу достаточно развит для кристаллов с различным типом упорядочения [27,28,32-35], в том числе для систем на основе $\mathrm{SrTiO}_{3}$ с примесями $[17,18]$. Из рис. 6 видно, что, температурный ход $\delta \Psi(T)$ в области высоких температур для монокристаллов SCT-1.4 очень близок к поведению $\delta \Psi(T)$ в STO. Учитывая это, для определения $\delta \Psi^{0}(T)$ в SCT-1.4 мы использовали экстраполяцию температурного хода $\delta \Psi(T)$ из высокотемпературной параобласти в область низких температур по реперной зависимости $\delta \Psi_{r}^{0}(T)$, рассчитанной на основе температурных изменений $\delta \Psi_{a}(T)$ (кривая 3 на рис. 6) в номинально чистом титанате стронция. Выбор $\delta \Psi_{a}(T)$ для расчета реперной зависимости $\delta \Psi_{r}^{0}(T)$ был обусловлен тем, что в STO ниже $T_{0}=105 \mathrm{~K}$ вклад параметра порядка структурного фазового перехода в $\delta \Psi_{a}(T)$ меньше, чем в $\delta \Psi_{c}(T)$. Пример такой экстраполяции температурной зависимости регулярного вклада $\delta \Psi_{a}^{0}(T)$ для SCT-1.4 приведен на вставке рис. 6 (кривая 5). Разность между экспериментальной зависимостью $\delta \Psi_{a}(T)$ (кривая 1 на 
вставке рис. 6) и расчетной для регулярного вклада $\delta \Psi_{a}^{0}(T)$ (кривая 5) представляет собой спонтанный полярный вклад в рефракцию света $\delta \Psi_{a}^{s}(T)$. Температурные зависимости $\delta \Psi_{i}^{s}(T)$, полученные таким образом для SCT-1.4, приведены на рис. 8. Во всех исследованных образцах полярный вклад $\delta \Psi_{i}^{s}(T)$ возникает при температурах существенно больших температуры Кюри, что очевидно связано с флуктуациями поляризации (ближним, локальным полярным порядком). На этом же рисунке приведена температурная зависимость морфического двупреломления $\Delta n_{a b}$. Под морфическим двупреломлением здесь и далее понимается двупреломление, обусловленное изменением симметрии кристалла при фазовом переходе. (В данном случае это полярное морфическое двупреломдение, возникающее при сегнетоэлектрическом переходе). В отличие от полярного вклада в рефракцию света $\delta \Psi_{a}^{s}(T)$ и $\delta \Psi_{c}^{s}(T) \Delta n_{a b}$ выше $T_{c}$ отсутствует. Это означает, что флуктуации поляризации изотропны вдоль осей $a$ и $b$ образца, по крайней мере выше $T_{c}$.

\section{4. Вычисление спонтанной поляризации и ее флуктуаций из рефрактометрических измерений}

В нашей работе [25] в рамках феноменологического подхода показано, что для актуальных полярных групп симметрии 4mm, mm2 и ряда других спонтанный полярный вклад в рефракцию света, обусловленный квадратичным электрооптическим эффектом при появлении поляризации и ее флуктуаций, для главных значений показателя преломления дается выражением

$$
\delta n_{i}^{s}=-\sum_{k=1}^{3}\left(\frac{n_{i}^{3}}{2}\right) g_{i k}^{*}\left(\left\langle P_{s k}^{2}\right\rangle+\left\langle P_{\mathrm{fl} k}^{2}\right\rangle\right),
$$

где $P_{s k}=\left|\left\langle P_{s k}\right\rangle\right|$ - абсолютные значения компонент спонтанной поляризации, связанные с дальним порядком, а $P_{\mathrm{flk}}$ - абсолютные значения флуктуационных компонент спонтанной поляризации, которые связаны только с ближним (локальным) порядком, $g_{i k}^{*}-$ квадратичные по поляризации электрооптические (ЭО) коэффициенты в полярной фазе. Для монодоменного кристалла, когда спонтанная поляризация направлена вдоль оси, обозначенной индексом 3, используя обозначения $P_{s}=\left|\left\langle P_{s 3}\right\rangle\right|$ и $P_{\mathrm{sh} k}=\left\langle P_{\mathrm{fl} k}^{2}\right\rangle^{1 / 2}$, получаем систему из трех уравнений $[25,26]$ :

$$
\begin{aligned}
& \delta n_{1}^{s}=-\frac{n_{1}^{3}}{2}\left[g_{13}^{*}\left(P_{s}^{2}+P_{\mathrm{sh} 3}^{2}\right)+g_{11}^{*} P_{\mathrm{sh} 1}^{2}+g_{12}^{*} P_{\mathrm{sh} 2}^{2}\right], \\
& \delta n_{2}^{s}=-\frac{n_{2}^{3}}{2}\left[g_{23}^{*}\left(P_{s}^{2}+P_{\mathrm{sh} 3}^{2}\right)+g_{21}^{*} P_{\mathrm{sh} 1}^{2}+g_{22}^{*} P_{\mathrm{sh} 2}^{2}\right], \\
& \delta n_{3}^{s}=-\frac{n_{3}^{3}}{2}\left[g_{33}^{*}\left(P_{s}^{2}+P_{\mathrm{sh} 3}^{2}\right)+g_{31}^{*} P_{\mathrm{sh} 1}^{2}+g_{32}^{*} P_{\mathrm{sh} 2}^{2}\right] .
\end{aligned}
$$

Выражения (3) являются системой из 3-х уравнений с четырьмя неизвестными. Измерения температурных изменений трех главных значений показателя преломления в монодоменном кристалле $\delta n_{i}(T)$, после вычитания регулярного вклада $\delta n_{i}^{0}(T)$ (не связанного с поляризацией) и выделения $\delta n_{i}^{s}(T)$, позволяют на основе системы (3) определить величину и температурную зависимость $P_{\text {sh }}$ выше и ниже температуры Кюри, если из независимых измерений определена величина $P_{s}$, а также получать $P_{\text {sh }}$ в системах с локальным полярным упорядочением (без дальнего порядка). Конечно, если известны ЭО-коэффициенты. (Как правило, квадратичные по поляризации электрооптические коэффициенты слабо зависят от температуры [36].) В случае изотропных флуктуаций для определения $P_{s}$ и $P_{\mathrm{sh}}$ достаточно двух уравнений из системы (3). Для анизотропных флуктуаций из (3) определяется сумма $\left(P_{s}^{2}+P_{\mathrm{sh} 3}^{2}\right)$. В этом случае конечно необходимы измерения $P_{s}$ другими независимыми методами, например, из петель диэлектрического гистерезиса.

При интерферометрических измерениях спонтанный полярный вклад в относительные изменения оптического пути света, полученный после вычитания из экспериментально измеренной зависимости $\delta \Psi_{i}(T)$ регулярного вклада $\delta \Psi_{i}^{0}(T)$, не связанного с поляризацией, дается выражением

$$
\delta \Psi_{i}^{s}=\delta n_{i}^{s}+\left(n_{i}-1\right) \frac{\delta l_{j}^{s}}{l_{j}},
$$

где $\delta n_{i}^{s}$ является спонтанным полярным вкладом в главные значения показателя преломления и определяется выражением (2), а $\delta l_{j}^{s}-$ спонтанный вклад в тепловое расширение, возникающий за счет спонтанной стрикции. Тогда спонтанный полярный вклад $\delta \Psi_{i}^{s}(T)$ дается выражением $[25,26]$ :

$$
\begin{aligned}
\delta \Psi_{i}^{s} & =\sum_{k=1}^{3}\left(-\frac{n_{i}^{3}}{2} g_{i k}^{*}+\left(n_{i}-1\right) Q_{j k}^{*}\right)\left\langle P_{k}^{2}\right\rangle \\
& =-\sum_{k=1}^{3}\left(\frac{n_{i}^{3}}{2}\right)\left[g_{i k}^{*}-\frac{2}{n_{i}^{3}}\left(n_{i}-1\right) Q_{j k}^{*}\right]\left\langle P_{k}^{2}\right\rangle .
\end{aligned}
$$

Вводя эффективные электрооптические коэффициенты

$$
g_{i k}^{* *}=\left[g_{i k}^{*}-\frac{2}{n_{i}^{3}}\left(n_{i}-1\right) Q_{j k}^{*}\right]
$$

и используя те же обозначения, что при записи системы (3), для $\delta \Psi_{i}^{s}(T)$ получим систему уравнений $[25,26]$, аналогичную (3):

$$
\begin{aligned}
& \delta \Psi_{1}^{s}=-\frac{n_{1}^{3}}{2}\left[g_{13}^{* *}\left(P_{s}^{2}+P_{\mathrm{sh} 3}^{2}\right)+g_{11}^{* *} P_{\mathrm{sh} 1}^{2}+g_{12}^{* *} P_{\mathrm{sh} 2}^{2}\right], \\
& \delta \Psi_{2}^{s}=-\frac{n_{2}^{3}}{2}\left[g_{23}^{* *}\left(P_{s}^{2}+P_{\mathrm{sh} 3}^{2}\right)+g_{21}^{* *} P_{\mathrm{sh} 1}^{2}+g_{22}^{* *} P_{\mathrm{sh} 2}^{2}\right], \\
& \delta \Psi_{3}^{s}=-\frac{n_{3}^{3}}{2}\left[g_{33}^{* *}\left(P_{s}^{2}+P_{\mathrm{sh} 3}^{2}\right)+g_{31}^{* *} P_{\mathrm{sh} 1}^{2}+g_{32}^{* *} P_{\mathrm{sh} 2}^{2}\right] .
\end{aligned}
$$


В $[25,26,37]$ также показано, что при интерферометрических исследованиях ЭО-коэффициентов измеряются именно эффективные коэффициенты $g_{i k}^{* *}$, входящие в (6).

Таким образом, $P_{\mathrm{sh}}$, а случае изотропных флуктуаций и $P_{s}$, и $P_{\text {sh }}$ могут быть определены из интерферометрических изменений $\delta \Psi_{i}(T)$ (после выделения $\delta \Psi_{i}^{s}$ ) и $\delta \Psi_{i}(E)$ без измерений теплового расширения, входящего в формулу (1). Поэтому, используя термин рефракция света, мы имеем ввиду оба вклада в $\delta \Psi_{i}(T)$ (от показателя преломления и от расширения).

\section{5. Симметрия сегнетоэлектрической фазы в твердом растворе $\mathrm{Sr}_{1-x} \mathrm{Ca}_{x} \mathrm{TiO}_{3}$}

При использовании системы (6) для вычисления величины и температурной зависимости $P_{\text {sh }}$ из экспериментальных данных $\delta \Psi_{i}(T)$ в SCT принципиальную роль играет корректное определение направления спонтанной поляризации $\mathbf{P}_{s}$ ниже $T_{c}$ : вдоль или поперек оси 4-го порядка. В АФД-фазе мягкая ТО-мода $T_{1 u}$ расщепляется на две моды $A_{2 u}$ и $E_{u}$, соответствующие смещениям ионов вдоль и поперек оси $C_{4}$ [2]. Обе моды сохраняют устойчивость вплоть до $T=0 \mathrm{~K}$, благодаря как вкладу энергии нулевых колебаний в полную энергию, так и положительному сдвигу обеих мод относительно моды $T_{1 u}$ вследствие тетрагонального искажения решетки в АФД-фазе $[1,3]$. Наличие СЭ-перехода в твердом растворе SCT с зависящей от $x$ температурой Кюри $T_{c}(x)$ демонстрирует увеличение классического вклада дестабилизирующих сил в ИК-активные ТО-моды с ростом $x$ [31]. СЭ-свойства твердого раствора типа смещения SCT [31] можно понять с помощью метода виртуального кристалла $(1-x) \mathrm{SrTiO}_{3}-x \mathrm{CaTiO}_{3}$, используя результаты расчетов $a b$ initio для компонент твердого раствора [31]. Для STO расчеты $a b$ initio классического вклада в частоты $\omega_{c} \equiv \omega\left(A_{2 u}\right)$ и $\omega_{a} \equiv \omega\left(E_{u}\right)$ мод $A_{2 u}$ и $E_{u}$ предсказывают СЭ-неустойчивость АФД-фазы в классическом приближении [38,39], причем более неустойчивой оказывается $E_{u}$ мода, соответствующая направлению $\mathbf{P}_{s}$ вдоль одной из осей 2-го порядка в плоскости, перпендикулярной оси $C_{4}$. Ввиду некоторого расхождения в результатах этих работ, мы выполнили расчеты $a b$ initio полных энергий основного состояния в классическом приближении для трех низкотемпературных структурных фаз STO: неполярной тетрагональной AFD-фазы с симметрией $I 4 / m c m-D_{4 h}^{18}$ (пр. группа 140), тетрагональной СЭ-фазы с симметрией $I 4 \mathrm{~cm}-C_{4 v}^{10}$ (пр. группа 108) с $\mathbf{P}_{s}$ вдоль оси $C_{4}$ (L्L-фаза) и ромбической СЭ-фазы Ima2- $C_{2 v}^{22}$ (пр. группа 46) с $\mathbf{P}_{s}$ вдоль оси $C_{2}$ (T्Т-фаза). Все расчеты выполнены в базисе плоских волн $(\mathrm{PW})$ и основаны на теории функционала плотности (DFT) в рамках обобщенного градиентного приближения (GGA) с использованием сверхмягких атомных псевдопотенциалов $a b$ initio (Sr.pbe-nsp-van.UPF, Ca.pbe-nsp-van.UPF, Ti.pbe-sp-van_ak.UPF, O.pbe-van_ak.UPF [40]). Использован обменно-корреляционный функционал ПедьюБурке-Ернцерхофа (PBE) [41] типа GGA, включающий как локальные, так и нелокальные обменный и корреляционный вклады. Использован набор $k$-узлов в зоне Бриллюэна, эквивалентный $8 \times 8 \times 8$ сетке МонкхорстаПака [42], а также энергия обрезания $100 \mathrm{Ry}$. Bce расчеты выполнены с использованием кода Quantum Espresso (Ver. 6.1) [43]. Расчеты выигрышей в энергии $\Delta E_{\text {min }}$ для $\mathscr{L}$-фазы и $\mathscr{T - ф а з ы ~ о т н о с и т е л ь н о ~ А Ф Д - ф а з ы ~}$ дают: $\Delta E_{\min }(\mathscr{L})=7.6 \mathrm{meV}$ и $\Delta_{\min }(\mathscr{T})=11.4 \mathrm{meV}$ при расщеплении $3.8 \mathrm{meV}$. Эти результаты получены для полностью оптимизированной структуры всех трех фаз. Таким образом, наши расчеты показывают, что в STO в классическом приближении основным состоянием является $\mathscr{T - ф а з а ~ с ~ п о л я р и з а ц и е и ̆ ~} \mathbf{P}_{s}$, направленной вдоль одной из осей 2-го порядка. Аналогичные расчеты для фаз той же симметрии были выполнены для $\mathrm{CaTiO}_{3}$ (CТО). Было найдено, что для СТО $\Delta E_{\min }(\mathscr{L})=0.0 \mathrm{meV}$ и $\Delta E_{\min }(\mathscr{T})=5.8 \mathrm{meV}$. Нулевые значения $\Delta E_{\min }(\mathscr{L})$ означают устойчивость АФД-фазы относительно возникновения $\mathscr{L}$-фазы. Структура АФД-фазы не является основным состоянием в СТО и, согласно нашим расчетам, лежит на $94 \mathrm{meV}$ выше основного состояния СТО. Однако наблюдаемое в SCT при $x \ll 1$ значительное повышение $T_{0}$ с ростом $x$ свидетельствует, на наш взгляд, о доминировании этой структуры при параметрах решетки, соответствующих STO. Для СTO и, в соответствии с моделью виртуального кристалла, для SCT в этой области параметров решетки можно ожидать увеличения выигрыша в энергии $\Delta E_{\min }(\mathscr{T})$ для полярной $T$-фазы, превышающего вклад нулевых колебаний, и формирования ромбической СЭ-структуры со спонтанной поляризаций $\mathbf{P}_{s}$, направленной вдоль одной из осей $C_{2}$. Таким образом, наши результаты показывают, что как в STO, так и в твердом растворе $(1-x) \mathrm{SrTiO}_{3}-x \mathrm{CaTiO}_{3}$ в качестве основного состояния в классическом приближении следует ожидать ромбическую СЭ-фазу с симметрией $\operatorname{Ima2-} C_{2 v}^{22}$ (пр. группа 46) c $\mathbf{P}_{s}$ вдоль одной из осей $C_{2}$, ортогональной главной оси четвертого порядка АФД-структуры (рис. 1).

\section{6. Характеристики индуцированной сегнетофазы в $\mathrm{Sr}_{1-x} \mathrm{Ca}_{x} \mathrm{TiO}_{3}$}

Для вычисления $P_{s}$ и $P_{\mathrm{sh}}$ в $\mathrm{SCT}-1.4$ из выделенного спонтанного вклада в рефракцию света $\delta \Psi_{a}^{s}(T)$ и $\delta \Psi_{c}^{s}(T)$ и морфического двупреломления $\Delta n_{a b}$ из системы уравнений (6) необходимо знание электрооптических коэффициентов $g_{i k}^{* *}$ в сегнетофазе. В нашей работе [37] установлено, что при комнатной температуpe ЭО-коэффициенты SCT-1.4 в пределах погрешности 
измерений совпадают с ЭО-коэффициентами STO [44]. Следует отметить, что в $[37,44]$ измерялись именно эффективные в указанном выше понимании ЭО-коэффициенты в параэлектрической фазе, для которых в литературе, как правило, используются обозначения $g_{i k}$. Учитывая это, далее в параэлектрической фазе будем следовать общепринятым обозначениям $g_{i k}$ без звездочки, понимая под ними эффективные ЭО-коэффициенты. В кубической центросимметричной парафазе STO и SCT ЭО-эффект описывается тремя независимыми коэффициентами $g_{11}, g_{12}, g_{44}$. Для кислородно-октаэдрических сегнетоэлектриков ЭО-коэффициенты в полярной фазе могут быть выражены через ЭО-коэффициенты в параэлектрической фазе [23,24]. Это справедливо и для эффективных ЭО-коэффициентов $[25,26]$. В низкотемпературной полярной фазе $\mathrm{Sr}_{1-x} \mathrm{Ca}_{x} \mathrm{TiO}_{3}$ симметрии $m m 2\left(C_{2 v}\right)[10,14,20,21] g_{i k}^{* *}$ и $g_{11}, g_{12}, g_{44}$ связаны соотношениями $[17,18,26]$ :

$$
\begin{gathered}
g_{13}^{* *}=g_{12}, \quad g_{23}^{* *}=1 / 2\left(g_{11}+g_{12}-g_{44}\right), \\
g_{33}^{* *}=1 / 2\left(g_{11}+g_{12}+g_{44}\right) .
\end{gathered}
$$

При расчетах $P_{s}$ и $P_{\mathrm{sh}}$ для SCT-1.4 из спонтанных вкладов $\delta \Psi_{i}^{s}$ по уравнениям (6) были использованы значения ЭО-коэффициентов $g_{11}=0.15 \mathrm{~m}^{4} / \mathrm{C}^{2}$, $g_{12}=0.04 \mathrm{~m}^{4} / \mathrm{C}^{2}, g_{44}=0.08 \mathrm{~m}^{4} / \mathrm{C}^{2}$ и показателя преломления $n_{i}=n=2.39$. В работе [45] показано, что ЭО-коэффициенты номинально чистого титаната стронция практически не зависят от температуры. Аналогичное поведение мы предположили и для SCT-1.4.

Как следует из расчетов раздела 5 , в структурно монодоменном образце $\mathrm{Sr}_{1-x} \mathrm{Ca}_{x} \mathrm{TiO}_{3}$ в тетрагональной парафазе с осью 4-го порядка $[001]_{c}$ (ось $c$ ) при переходе в СЭ-фазу $\mathrm{Sr}_{1-x} \mathrm{Ca}_{x} \mathrm{TiO}_{3}$ симметрии $m m 2\left(C_{2 v}\right)$ спонтанная поляризация $P_{s}$ может лежать вдоль осей $[110]_{a}$ или $[1 \overline{1} 0]_{b}$ (рис. 1 ), что согласуется с интерпретацией экспериментальных данных в работах $[10,14,20,21,46]$. Поэтому возможны два варианта расчетов из уравнений (6): для монодоменного образца с $P_{s}=P_{s a}$ (поляризация вдоль направления $[110]_{a}$ ) и для монодоменного образца с $P_{s}=P_{s b}$ (поляризация вдоль направления $[1 \overline{1} 0]_{b}$ ). Для каждого из фиксированных направлений $P_{s}$ возможны флуктуации вдоль полярных осей $[110]_{a}$ и $[1 \overline{1} 0]_{b}\left(P_{\mathrm{sh} a}=\left\langle P_{\mathrm{fla}}^{2}\right\rangle^{1 / 2}\right.$ и $\left.P_{\mathrm{sh} b}=\left\langle P_{\mathrm{fl} b}^{2}\right\rangle^{1 / 2}\right)$. При конкретизации уравнений (6) далее будем использовать обозначения $P_{s}=\left|\left\langle P_{s 3}\right\rangle\right|=P_{s a}$ для спонтанной поляризации вдоль оси $[110]_{a}$ в монодоменном образце и $P_{s}=\left|\left\langle P_{s 3}\right\rangle\right|=P_{s b}$ для спонтанной поляризации вдоль оси $[1 \overline{1} 0]_{b}$. С целью упрощения уравнений для расчетов по каждому из этих вариантов ориентации $P_{s}$ в SCT обозначим $A_{i 3}=-\left(n^{3} / 2\right) g_{i 3}^{* *}$. Тогда, учитывая соотношения (7), численные значения $g_{11}, g_{12}, g_{44}$ и $n$, получим

$$
\begin{gathered}
A_{13}=-\left(n^{3} / 2\right)\left(g_{12}\right)=-0.27\left[\mathrm{~m}^{4} / \mathrm{C}^{2}\right], \\
A_{23}=-\left(n^{3} / 4\right)\left(g_{11}+g_{12}-g_{44}\right)=-0.31\left[\mathrm{~m}^{4} / \mathrm{C}^{2}\right], \\
A_{33}=-\left(n^{3} / 4\right)\left(g_{11}+g_{12}+g_{44}\right)=-0.92\left[\mathrm{~m}^{4} / \mathrm{C}^{2}\right] .
\end{gathered}
$$

Из условий симметрии $A_{13}=A_{12}$ и $A_{23}=A_{32}$.
В этом случае варианты записи уравнений (6) будут иметь вид: $P_{s}=P_{s a}$ лежит вдоль оси $[110]_{a}$, флуктуации соответственно вдоль осей $[110]_{a}$ и $[1 \overline{10}]_{b}$.

$$
\begin{aligned}
\delta \Psi_{a}^{S}(T) & =A_{33} P_{s a}^{2}+A_{33} P_{\mathrm{sh} a}^{2}+A_{23} P_{\mathrm{sh} b}^{2} \\
& =A_{33}\left(P_{s a}^{2}+P_{\mathrm{sh} a}^{2}\right)+A_{23} P_{\mathrm{sh} b}^{2}, \\
\delta \Psi_{b}^{S}(T) & =A_{23} P_{s a}^{2}+A_{23} P_{\mathrm{sh} a}^{2}+A_{33} P_{\mathrm{sh} b}^{2} \\
& =A_{23}\left(P_{s a}^{2}+P_{\mathrm{sh} a}^{2}\right)+A_{33} P_{\mathrm{sh} b}^{2}, \\
\delta \Psi_{c}^{S}(T) & =A_{13} P_{s a}^{2}+A_{13} P_{\mathrm{sh} a}^{2}+A_{13} P_{\mathrm{sh} b}^{2} \\
& =A_{13}\left(P_{s a}^{2}+P_{\mathrm{sh} a}^{2}+P_{\mathrm{sh} b}^{2}\right) .
\end{aligned}
$$

Для морфического двупреломления $\Delta n_{a b}=n_{b}-n_{a}$

$$
\begin{aligned}
\Delta n_{a b}=\delta n_{b}^{s} & -\delta n_{a}^{s}=\delta \Psi_{b}^{S}-\delta \Psi_{a}^{S}=\left(A_{23}-A_{33}\right) P_{s a}^{2} \\
& +\left(A_{23}-A_{33}\right) P_{\text {sh } a}^{2}-\left(A_{23}-A_{33}\right) P_{\text {sh } b}^{2} .
\end{aligned}
$$

Согласно нашим измерениям морфическое двупреломление $\Delta n_{a b}$ в SCT-1.4 выше $T_{c}$ равно нулю (рис. 8). Из (9) следует, что в этом случае $P_{\mathrm{sh} a}^{2}=P_{\mathrm{sh} b}^{2}$ выше $T_{c}$. Можно предположить, что флуктуации поляризации вдоль осей $[110]_{a}$ и $[1 \overline{1} 0]_{b}$ изотропны и ниже $T_{c}$ и $P_{\mathrm{sh} a}^{2}=P_{\mathrm{sh} b}^{2}$. При $P_{\mathrm{sh}}^{2}=P_{\mathrm{sh} a}^{2}=P_{\mathrm{sh} b}^{2}$ и подстановки численных значений коэффициентов уравнения (8) будут иметь вид

$$
\begin{aligned}
\delta \Psi_{a}^{S}(T) & =A_{33} P_{s a}^{2}+\left(A_{33}+A_{23}\right) P_{\mathrm{sh}}^{2} \\
& =\left[-0.92 P_{s a}^{2}-1.23 P_{\mathrm{sh}}^{2}\right], \\
\delta \Psi_{b}^{S}(T) & =A_{23} P_{s a}^{2}+\left(A_{23}+A_{33}\right) P_{\mathrm{sh}}^{2} \\
& =\left[-0.31 P_{s a}^{2}-1.23 P_{\mathrm{sh}}^{2}\right], \\
\delta \Psi_{c}^{S}(T)=A_{13} P_{s a}^{2} & +2 A_{13} P_{\mathrm{sh}}^{2}=\left[-0.27 P_{s a}^{2}-0.54 P_{\mathrm{sh}}^{2}\right]
\end{aligned}
$$

спонтанный полярный вклад в кристаллографическое двупреломление

$$
\begin{aligned}
\Delta n_{a c}^{s}= & \delta \Psi_{c}^{S}(T)-\delta \Psi_{a}^{S}(T)=\left(A_{13}-A_{33}\right) P_{s a}^{2} \\
& +\left(2 A_{13}-A_{33}-A_{23}\right) P_{\mathrm{sh}}^{2}=\left[0.65 P_{s a}^{2}-0.69 P_{\mathrm{sh}}^{2}\right],
\end{aligned}
$$

морфическое двупреломление $\Delta n_{a b}=n_{b}-n_{a}$ :

$$
\begin{aligned}
\Delta n_{a b} & =\delta n_{b}^{s}-\delta n_{a}^{s}=\delta \Psi_{b}^{S}-\delta \Psi_{a}^{S} \\
& =\left(A_{23}-A_{33}\right) P_{s a}^{2}=\left[+0.61 P_{s a}^{2}\right] .
\end{aligned}
$$

Для случая, когда $P_{s}=P_{s b}$ лежит вдоль оси $[1 \overline{1} 0]_{b}$ и флуктуации вдоль осей $[110]_{a}$ и $[1 \overline{1} 0]_{b}$ изотропны $P_{\mathrm{sh}}^{2}=P_{\mathrm{sh} a}^{2}=P_{\mathrm{sh} b}^{2}$ выражения (6) для $\delta \Psi_{i}^{S}(T)$ запишутся как

$$
\begin{aligned}
\delta \Psi_{a}^{S}(T) & =A_{23} P_{s b}^{2}+\left(A_{23}+A_{33}\right) P_{\mathrm{sh}}^{2} \\
& =\left[-0.31 P_{s b}^{2}-1.23 P_{\mathrm{sh}}^{2}\right],
\end{aligned}
$$




$$
\begin{gathered}
\delta \Psi_{b}^{S}(T)=A_{33} P_{s b}^{2}+\left(A_{33}+A_{23}\right) P_{\mathrm{sh}}^{2} \\
=\left[-0.92 P_{s b}^{2}-1.23 P_{\mathrm{sh}}^{2}\right], \\
\delta \Psi_{c}^{S}=A_{13} P_{s b}^{2}+2 A_{13} P_{\mathrm{sh}}^{2}=\left[-0.27 P_{s b}^{2}-0.54 P_{\mathrm{sh}}^{2}\right],
\end{gathered}
$$

спонтанный полярный вклад в кристаллографическое двупреломление

$$
\begin{aligned}
\Delta n_{a c}^{s}= & \delta \Psi_{c}^{S}(T)-\delta \Psi_{a}^{S}(T)=\left(A_{13}-A_{23}\right) P_{s b}^{2} \\
& +\left(2 A_{13}-A_{33}-A_{23}\right) P_{\mathrm{sh}}^{2}=\left[0.04 P_{s b}^{2}-0.69 P_{\mathrm{sh}}^{2}\right],
\end{aligned}
$$

морфическое двупреломление $\Delta n_{a b}=n_{b}-n_{a}$ :

$$
\begin{aligned}
\Delta n_{a b} & =\delta n_{b}^{s}-\delta n_{a}^{s} \\
& =\delta \Psi_{b}^{S}-\delta \Psi_{a}^{S}=\left(A_{33}-A_{23}\right) P_{s b}^{2}=\left[-0.61 P_{s b}^{2}\right] .
\end{aligned}
$$

В результате получаем две серии уравнений для двух возможных вариантов монодоменного кристалла $(10)-(14)$ для $P_{s a}$ и $(15)-(19)$ для $P_{s b}$. В предположении изотропных флуктуаций для каждого варианта экспериментальных данных хватает с избытком, чтобы вычислить и $P_{s}$, и $P_{\mathrm{sh}}$ только из оптических измерений. Набор экспериментальных данных позволяет провести расчеты для двух вариантов направления спонтанной поляризации монодоменного кристалла $\left(P_{s a}\right.$ и $\left.P_{s b}\right)$ и тем самым уточнить преимущественное направление $P_{s}\left(P_{s a}\right.$ или $\left.P_{s b}\right)$. Учитывая большую точность измерения двупреломления, при решении систем уравнений $(10)-(14)$ и $(15)-(19) P_{s}\left(P_{s a}\right.$ и $\left.P_{s b}\right)$ рассчитывалось из морфического двупреломления (14) и (19), а затем, после подстановки $P_{s}$ в $(10),(12)$ и соответственно в $(15),(17)$ определялось $P_{\mathrm{sh}}$ для обеих вариантов. Сравнение расчетов из $\delta \Psi_{a}^{S}(T)$ и $\delta \Psi_{c}^{S}(T)$ и морфического двупреломления $\Delta n_{a b}$ для каждого возможного варианта реализации монодоменного кристалла (со спонтанной поляризацией $P_{s b}$ или $\left.P_{s a}\right)$ позволяет провести верификацию направления спонтанной поляризации и в целом оценить правильность подхода. Значения $P_{s}=P_{s \text { LOOPS, }}$ получаемые из петель диэлектрического гистерезиса, являются проверочными данными для реалистичности

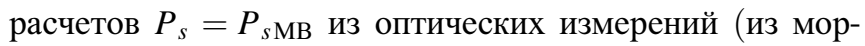
фического двупреломления).

Результаты расчетов приведены на рис. 9, $a, b$ и рис. 10 .

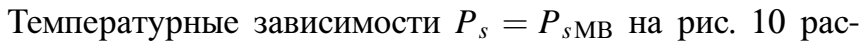
считаны по формулам (14), (19) из морфического двупреломления $\Delta n_{a b}(T)$, представленного на рис. 8. Полученные значения для $P_{s}=P_{s \mathrm{MB}}(T)$ и экспериментальные данные для $\delta \Psi_{a}^{S}(T)$ и $\delta \Psi_{c}^{S}(T)$ подставлены в уравнения $(10),(12)$ и $(15),(17)$, из которых для каждого $\delta \Psi_{a}^{S}(T)$ и $\delta \Psi_{c}^{S}(T)$ вычислены зависимости $P_{\mathrm{sh}}$ для обоих вариантов возможного направления спонтанной поляризации в образце SCT-1.4 (рис. 9, $a, b$ ). Видно, что зависимости $P_{\mathrm{sh}}(T)$, рассчитанные из $\delta \Psi_{a}^{S}(T)$ и $\delta \Psi_{c}^{S}(T)$, совпадают только для случая $P_{s}=P_{s b}$, когда спонтанная поляризация лежит вдоль оси $[1 \overline{1} 0]_{b}$. Это
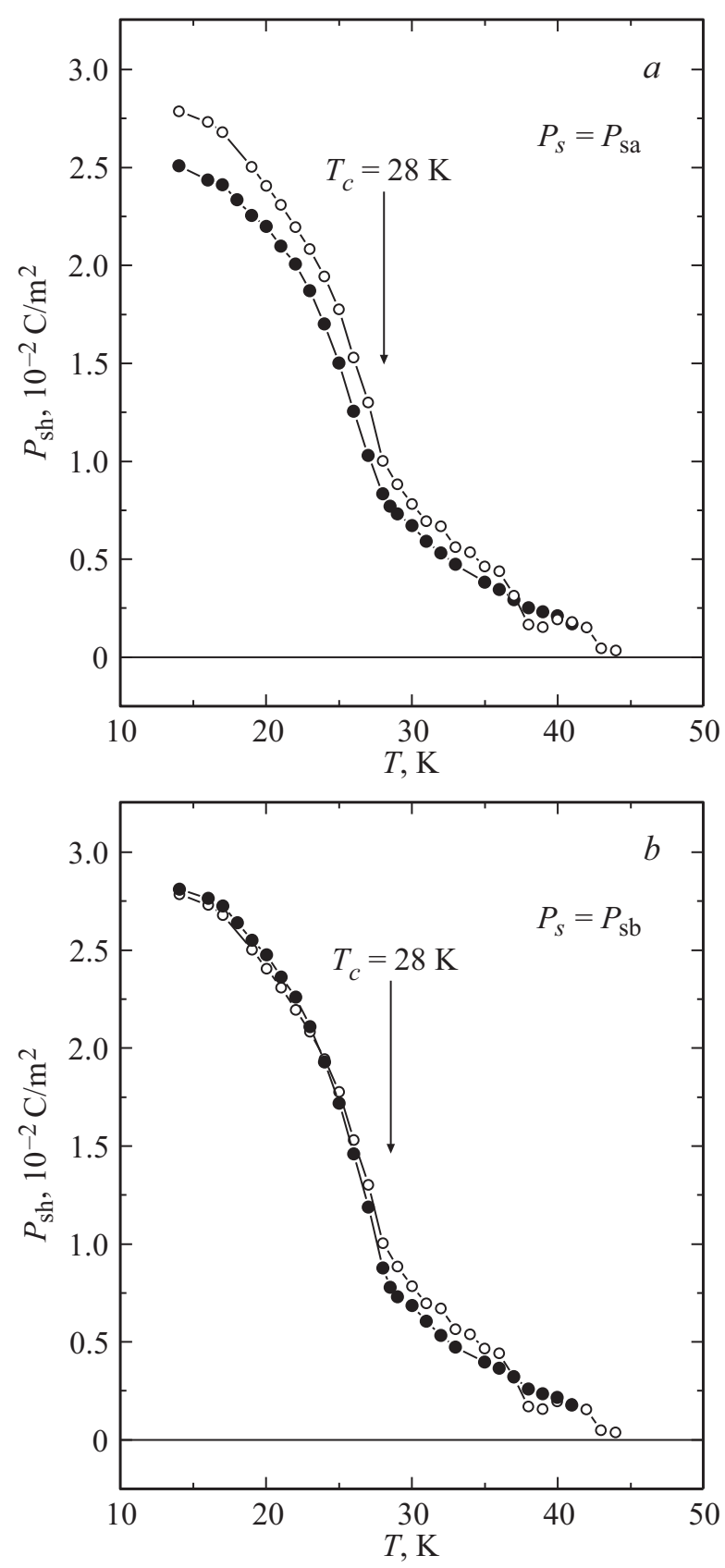

Pис. 9. $a-$ температурные зависимости $P_{\mathrm{sh}}$ в SCT-1.4, вычисленные из $\delta \Psi_{a}^{S}$ (точки) и $\delta \Psi_{c}^{S}$ (кружки) с использованием уравнений $(10),(12)$ для $P_{s}$, лежащей вдоль оси $[110]_{a}$ $\left(P_{s}=P_{s a}\right) . b-$ температурные зависимости $P_{\text {sh }}$ в SCT-1.4, вычисленные из $\delta \Psi_{a}^{S}$ (точки) и $\delta \Psi_{c}^{S}$ (кружки) с использованием уравнений $(15),(17)$ для $P_{s}$, лежащей вдоль оси $[1 \overline{1} 0]_{b}$ $\left(P_{s}=P_{s b}\right)$.

направление соответствует одной из легких осей ориентации спонтанной поляризации, полученных в наших расчетах, и согласующихся с интерпретацией экспериментов в работах $[10,14,20,21,46]$. Однако преимущественным направлением спонтанной поляризации $P_{s}$ при геометрии вырезки образцов SCT-1.4, использованной нами для формирования структурно монодоменного 


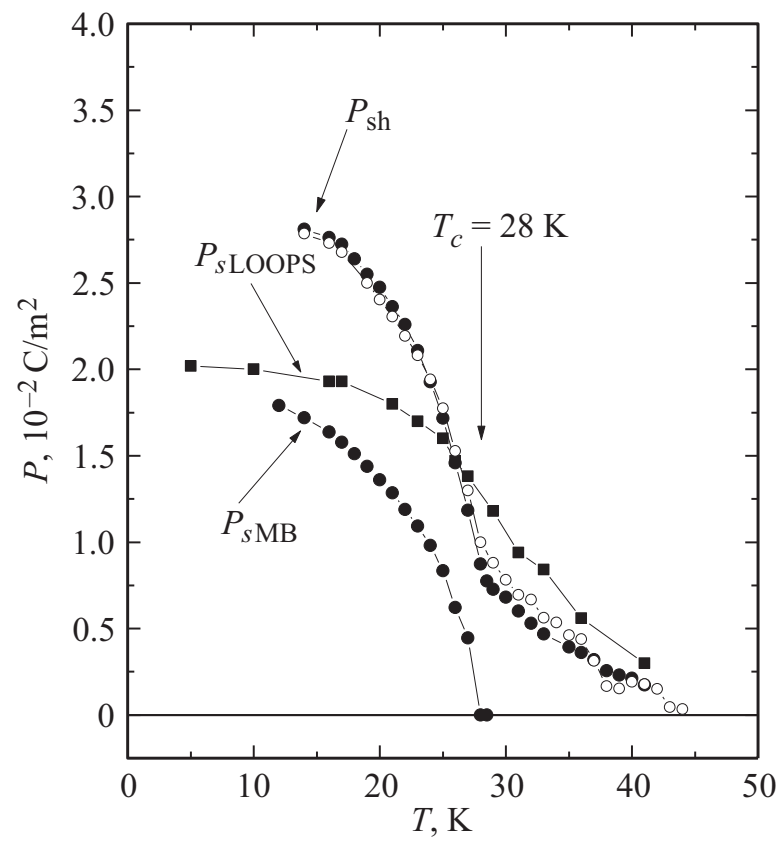

Рис. 10. Спонтанная поляризация, определенная из морфического двупреломления $P_{s \mathrm{MB}}$ и из петель диэлектрического гистерезиса $P_{s \text { LoOPS; }}$ зависимости $P_{\mathrm{sh}}(T)$, вычисленные из $\delta \Psi_{a}^{S}$ (точки) и $\delta \Psi_{c}^{S}$ (кружки) с использованием уравнений (15), (17) для случая $P_{s}$, лежащей вдоль оси $[1 \overline{1} 0]_{b}$ в SCT-1.4.

кристалла в АФД-фазе, оказывается направление вдоль оси $[1 \overline{1} 0]_{b}$ образца. Для $P_{s}=P_{s b}$ совпадение наблюдается в пределах 2\% (рис. 9, $b$ ) тогда как аналогичные кривые $P_{\mathrm{sh}}(T)$, рассчитанные из $\delta \Psi_{a}^{S}(T)$ и $\delta \Psi_{c}^{S}(T)$ для $P_{S}$, направленной вдоль оси $[110]_{a}$, отличаются более чем на $10 \%$ (рис. 9, $a$ ). Данные, представленные на рис. 10, и дальнейшее обсуждение относятся именно к случаю ориентации спонтанной поляризации вдоль оси $[1 \overline{1} 0]_{b}$. Обозначения $P_{s}$ и $P_{\mathrm{sh}}$ соответствуют абсолютным значениям величин. На рис. 10 для сравнения представлена также спонтанная поляризация, полученная из петель диэлектрического гисте-

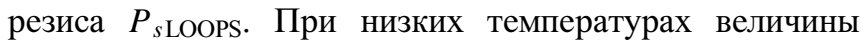
поляризации, полученной из морфического двупреломления $P_{s \mathrm{MB}}$ и петель гистерезиса $P_{s \text { LOOPS }}$ близки по

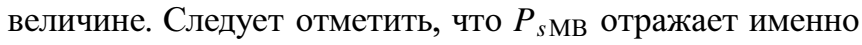
спонтанную поляризацию, тогда как $P_{s}$ LOOPS получено при воздействии на образец достаточно сильного электрического поля. Из рис. 10 видно, что в SCT-1.4 при $12 \mathrm{~K} P_{s}=P_{s \mathrm{MB}} \approx P_{s \text { LOOPS }} \approx 1.8 \cdot 10^{-2} \mathrm{C} / \mathrm{m}^{2}$, а $P_{\text {sh }}$ $\approx 2.7 \cdot 10^{-2} \mathrm{C} / \mathrm{m}^{2}$ при $14 \mathrm{~K}$. Большая величина $P_{\mathrm{sh}}$, превышающая величину $P_{s}$, указывает на важный вклад в формирование индуцированной примесями сегнетоэлектрической фазы в квантовых параэлектриках пространственных флуктуаций поляризации, которые по величине и температурной зависимости кардинально отличаются от классических термодинамических флуктуаций. В [21] поляризация $P_{s}$ была оценена при низких температурах для SCT $(x=0.007) \quad P_{s} \approx 2.3 \cdot 10^{-2} \mathrm{C} / \mathrm{m}^{2} \quad$ и $\quad$ в $\quad[22]$ для $\mathrm{SCT}$ $(x=0.011) P_{s} \approx 2.9 \cdot 10^{-2} \mathrm{C} / \mathrm{m}^{2}$ из спонтанного полярного вклада в кристаллографическое двупреломление. Эти величины существенно превосходят найденные нами значения $P_{s}=P_{s \text { LOOPS }} \approx 0.77 \cdot 10^{-2} \mathrm{C} / \mathrm{m}^{2}$ для $\mathrm{SCT}$ $(x=0.007) \quad$ и $P_{s}=P_{s \mathrm{MB}} \approx P_{s \mathrm{LOOPS}} \approx 1.8 \cdot 10^{-2} \mathrm{C} / \mathrm{m}^{2}$ для $\operatorname{SCT}(x=0.014)$, но близки по величине к найденному нами значению $P_{\mathrm{sh}} \approx 2.7 \cdot 10^{-2} \mathrm{C} / \mathrm{m}^{2}$. В особенности для SCT $(x=0.011)$, так как экстраполяция $P_{\mathrm{sh}}(T)$ к $T=0 \mathrm{~K}$ в SCT $(x=0.014)$ дает величину $P_{\mathrm{sh}}(0 \mathrm{~K}) \approx 3 \cdot 10^{-2} \mathrm{C} / \mathrm{m}^{2}$. Такие корреляции обусловлены тем, что в $[21,22]$ оценки проводились с использованием формул, которые не позволяют разделить вклады в рефракцию света среднего значения спонтанной поляризации $P_{s}$ и сильных пространственных флуктуаций поляризации $P_{\mathrm{sh}}$ (вклады ближнего и дальнего порядка), поэтому в поляризацию $P_{s}$, приведенную в $[21,22]$, вошел вклад $P_{\mathrm{sh}}$, величина которого ниже $T_{c}$ значительно превышает истинное значение спонтанной поляризации $P_{s}$, обусловленной дальним порядком (см. формулы (13), (18) для спонтанного полярного вклада в кристаллографическое двупреломление), т.е. оценки значения поляризации, полученные в [21,22], фактически были обусловлены величиной $P_{\mathrm{sh}}$.

Используя наш метод вычисления $P_{s}$ и $P_{\mathrm{sh}}$, на основе уравнений (18), (19) и экспериментальных данных, приведенных в работе $[14,20,21]$ по температурным зависимостям кристаллографического $\Delta n_{a c}$ и морфического $\Delta n_{a b}$ двупреломления мы оценили $P_{s}=P_{s \text { мв }}$ и $P_{\text {sh }}$ в $\mathrm{Sr}_{1-x} \mathrm{Ca}_{x} \mathrm{TiO}_{3}$ с $x=0.007$ при $T=5 \mathrm{~K}$. Оказалось, что при $T=5 \mathrm{~K}$ для $\mathrm{SCT}-0.7$ $P_{s \mathrm{MB}} \approx 0.7 \cdot 10^{-2} \mathrm{C} / \mathrm{m}^{2}$, что хорошо соответствует нашим данным для $P_{s}=P_{s \text { LOOPS }} \approx 0.77 \cdot 10^{-2} \mathrm{C} / \mathrm{m}^{2}$, полученным из петель гистерезиса в SCT-0.7 при той же температуре (рис. 5), а величина $P_{\text {sh }}$, характеризующая ближний (локальный) порядок, оказалась равной $P_{\mathrm{sh}} \approx 2.5 \cdot 10^{-2} \mathrm{C} / \mathrm{m}^{2}$. Таким образом, по меpe уменьшения концентрации примеси кальция, индуцирующей дальний сегнетоэлектрический порядок в $\mathrm{Sr}_{1-x} \mathrm{Ca}_{x} \mathrm{TiO}_{3}$, формирование дальнего порядка резко ослабляется. При уменьшении $x$ от 0.014 до $0.007 P_{s}$ уменьшается в 2.5 раза, а величина $P_{\mathrm{sh}}$, характеризующая локальный полярный порядок меняется слабо. Это указывает на то, что процессы формирования ближнего и дальнего порядка квазинезависимы и, вероятно, имеют разные механизмы.

Результаты $P_{s}(T)$ и $P_{\mathrm{sh}}(T)$, представленные на рис. 10 , на примере SCT впервые дают количественное описание формирования индуцированного примесями полярного состояния в квантовых параэлектриках. Значительно выше температуры $T_{c}$ возникают изотропные вдоль осей $a$ и $b\left(P_{\mathrm{sh}}^{2}=P_{a \mathrm{sh}}^{2}=P_{b \mathrm{sh}}^{2}\right)$ пространственные флуктуации поляризации. Величина $P_{\mathrm{sh}}$ возрастает по мере приближения к $T_{c}$, а скорость нарастания максимальна в $T_{c}$. Одновременно в $T_{c}$ появляется спонтанная поляризация вдоль оси $b$, величина которой сравнительно меньше 
пространственных флуктуаций. Дальнейшее охлаждение приводит к росту полярного порядка на обоих масштабах - дальнего и ближнего, $P_{s}$ и $P_{\mathrm{sh}}$. Таким образом происходит упорядочение на двух характерных масштабах: макроскопическом с дальним порядком и локальным упорядочением на нано- или мезоскопическом масштабе. Различные модельные представления такого упорядочения в системах квантовых параэлектриков с примесями и с изотопным замещением неоднократно обсуждались $[4-8,11,15]$, в том числе в системе $\mathrm{Sr}_{1-x} \mathrm{Ca}_{x} \mathrm{TiO}_{3}[7,14,21,22,30,46]$. Но количественных характеристик упорядочения на различных масштабах и их сравнений ранее не проводилось. Полученные данные позволили подтвердить ранее наблюдаемые особенности и выявить ряд новых. Результаты хорошо согласуются с предположением о том, что в данной геометрии изготовления образцов спонтанная поляризация направлена вдоль оси $b$. Наблюдается особенность (излом) в поведении $P_{\mathrm{sh}}$ при температуре СЭ-фазового перехода $\left(T_{c}=28 \mathrm{~K}\right)$, что указывает на наличие фазового перехода как на макроскопическом с поляризацией $P_{s}$ вдоль оси $b$, так и на нано- или мезоскопическом масштабе с локальным упорядочением вдоль осей $a$ и $b$. Таким образом, $P_{\text {sh }}$ можно рассматривать, как параметр порядка такого квазинезависимого процесса локального полярного упорядочения. Очевидно, что пространственные флуктуации, ответственные за появление $P_{\text {sh }}$, могут быть неоднородны по образцу. Величина $P_{\text {sh }}$ получена в результате усреднения по времени и объему образца квадрата локальной поляризации. В этом случае сегнетоэлектрическую фазу в $\mathrm{Sr}_{1-x} \mathrm{Ca}_{x} \mathrm{TiO}_{3}$ следует рассматривать как квазиоднородное полярное состояние с определенным средним значением макроскопической спонтанной поляризации $P_{s}$, возникающей на фоне локального полярного упорядочения, характеризуемого величиной $P_{\mathrm{sh}}$. В прин-

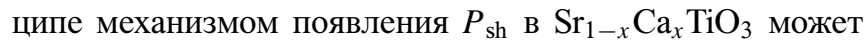
быть образование нанодоменной, кластерной структуры, обсуждаемой в работах $[14,21,30,46]$.

\section{7. Заключение}

В настоящей работе проведены термооптические и диэлектрические исследования сегнетоэлектрического фазового состояния в $\mathrm{Sr}_{1-x} \mathrm{Ca}_{x} \mathrm{TiO}_{3}$, индуцированного примесью кальция. Изучены температурные изменения рефракции света, двупреломления, диэлектрической проницаемости и петель диэлектрического гистерезиса в монокристаллах $\mathrm{Sr}_{1-x} \mathrm{Ca}_{x} \mathrm{TiO}_{3}$ с $x=0.014$ (SCT-1.4). В монокристаллах $\mathrm{Sr}_{1-x} \mathrm{Ca}_{x} \mathrm{TiO}_{3}$ с $x=0.007$ (SCT-0.7) проведены измерения температурных зависимостей диэлектрической проницаемости и петель диэлектрического гистерезиса. В SCT-1.4 выделен спонтанный полярный вклад в рефракцию света, возникающий за счет спонтанного квадратичного электрооптического эффекта, вызванного спонтанной поляризацией и ее флуктуациями. Выполнены расчеты $a b$ initio равновесных структур и полных энергий трех низкотемпературных фаз для $\mathrm{SrTiO}_{3}$ и $\mathrm{CaTiO}_{3}$, на основании которых определена ожидаемая симметрия основного состояния их твердого раствора и направления спонтанной поляризации в индуцированной кальцием сегнетофазе в $\mathrm{Sr}_{1-x} \mathrm{Ca}_{x} \mathrm{TiO}_{3}$.

Из спонтанного вклада в рефракцию света на основе ранее развитого авторами фенологического подхода [25] впервые количественно определены величины и температурные зависимости среднеквадратичных флуктуаций параметра порядка - поляризации $P_{\mathrm{sh}}=\left\langle P_{\mathrm{fl}}^{2}\right\rangle^{1 / 2}$ (ближний, локальный полярный порядок) в сегнетоэлектрической фазе, ниже температуры индуцированного сегнетоэлектрического перехода $T_{c}$. Из морфического двупреломления и петель диэлектрического гистерезиса в SCT-1.4 определено среднее значение спонтанной поляризации $P_{s}$ (вклад дальнего порядка). Результаты расчетов $P_{\text {sh }}$ и $P_{s}$ из температурных изменений различных главных значений рефракции света и морфического двупреломления показывают, что преимущественным направлением спонтанной поляризации $P_{s}$ при геометрии вырезки образцов SCT-1.4, использованной для формирования структурно монодоменного кристалла в АФД-фазе, является направление вдоль оси $[1 \overline{1} 0]_{b}$ образца. На основе наших данных по измерению петель диэлектрического гистерезиса и литературных данных по двупреломлению проведены оценки величин $P_{\text {sh }}$ и $P_{s}$, характеризующих ближний и дальний порядок в сегнетофазе SCT-0.7.

Полученные результаты позволили выявить на количественном уровне новые особенности формирования индуцированной полярной фазы в $\mathrm{Sr}_{1-x} \mathrm{Ca}_{x} \mathrm{TiO}_{3}$. Пространственные флуктуации (ближний, локальный порядок) поляризации, возникающие значительно выше $T_{c}$, имеют особенность в $T_{c}$, продолжают возрастать в сегнетоэлектрической фазе и существенно превышают среднее значение спонтанной поляризации, характеризующей дольний прядок. По мере уменьшения концентрации примеси кальция, индуцирующей дальний сегнетоэлектрический порядок в $\mathrm{Sr}_{1-x} \mathrm{Ca}_{x} \mathrm{TiO}_{3}$, формирование дальнего порядка резко ослабляется (при уменьшении $x$ от 0.014 до $0.007 P_{s}$ уменьшается в 2.5 раза), а ближний (локальный) полярный порядок, характеризуемый величиной $P_{\mathrm{sh}}$, меняется слабо. Выявленные особенности поведения $P_{s}$ и $P_{\mathrm{sh}}$ указывают на то, что процессы формирования ближнего и дальнего порядка квазинезависимы и, вероятно, имеют разные механизмы.

Следует отметить, что полученные результаты по определению $P_{\mathrm{sh}}$ и $P_{s}$ в SCT-1.4 - пример применения развитого авторами подходя [25] по количественному вычислению из экспериментальных рефрактометрических данных раздельно вкладов ближнего $P_{\mathrm{sh}}$ и дальнего $P_{s}$ порядка в формирование полярной фазы в системах с сосуществованием обоих вкладов в упорядоченной фазе ниже $T_{c}$. До настоящей работы нам известны только публикации, в которых приводятся результаты определения $P_{\mathrm{sh}}=\left\langle P_{\mathrm{fl}}^{2}\right\rangle^{1 / 2}$ для случаев, когда спонтанная поляризация, характеризующая дальний порядок $P_{s}=0$. 
Авторы благодарят В. Клееманна и Дж.Г. Беднорца за предоставление высококачественных монокристаллов $\mathrm{SrTiO}_{3}$ и $\mathrm{Sr}_{1-x} \mathrm{Ca}_{x} \mathrm{TiO}_{3}$ с $x=0.014$ и 0.007 .

\section{Список литературы}

[1] О.Е. Квятковский. ФТТ 43, 1345 (2001).

[2] A. Yamanaka, M. Kataoka, Y. Inaba, B. Hehlen, E. Courtens. Europhys. Lett. 50, 688 (2000).

[3] O.E. Kvyatkovskii. Solid State Commun. 117, 455 (2001).

[4] B.E. Vugmeister, M.D. Glinchuk. Rev. Mod. Phys. 62, 993 (1990).

[5] U.T. Hoechli, K. Knorr, A. Loidl. Adv. Phys. 39, 405 (1990).

[6] M.D. Glinchuk, I.P. Bykov. Phase Transitions 40, 1 (1992).

7] W. Kleemann. Int. J. Mod. Phys. B 7, 2469 (1993).

[8] G.A. Samara. J. Phys.: Condens. Mater. 15, R367 (2003).

[9] M. Itoh, R. Wang, Y. Inaguma, T. Yamaguchi, Y-J. Shan, T. Nakamura. Phys. Rev. Lett. 82, 3540 (1999).

[10] J.G. Bednorz, K.A. Mueller. Phys. Rev. Lett. 52, 2289 (1984).

[11] R. Blinc, B. Zalar, V.V. Laguta, M. Jtoh. Phys. Rev. Lett. 94, 147601-1 (2005).

[12] С.А. Просандеев, В.А. Трепаков. ЖЭТФ 121, 489 (2002).

[13] V.A. Trepakov, M.E. Savinov, S.A. Prosandeev, S.E. Kapphan, L. Jastrabik, L.A. Boatner. Phys. Status Solidi C 2, 145 (2005).

[14] W. Kleemann, U. Bianchi, A. Buergel, M. Prasse, J. Dec. Phase Transitions 55, 57 (1995).

[15] W. Kleemann, F.J. Schäfer, D. Rytz. Phys. Rev. Lett. 54, 2038 (1985).

[16] T. Azuma, K. Iio, K. Yamanaka, T. Kyomen, R. Wang, M. Itoh. Ferroelectrics 304, 77 (2004).

[17] P.A. Markovin, W. Kleemann, R. Linder, V.V. Lemanov, O.Yu. Korshuniv, P.P. Syrnikov. J. Phys.: Condens. Mater. 8, 2377 (1996).

[18] М.Е. Гужва, В. Клееманн, В.В. Леманов, П.А. Марковин. ФTT 39, 704 (1997).

[19] K. Yamanaka, R. Wang, M. Itoh, K. Iio. J. Phys. Soc. Jpn. 70, 3213 (2001).

[20] U. Bianchi. PhD Thesis, Gerhard-Mercator-UniversitätGesamthochschule-Duisburg, Duisburg (1996). 159 p.

[21] W. Kleemann, A. Albertini, M. Kuss, R. Lindner. Ferroelectrics 203, 57 (1997).

[22] W. Kleemann, F.J. Schafer, K.A. Miiller, J.G. Bednorz. Ferroelectrics 80, 297 (1988).

[23] M. DiDomeniko, S.H. Wemple. J. Appl. Phys. 40, 720 (1969).

[24] S.H. Wemple, M. DiDomenico. In: Applied Solid State Science / Ed. R. Wolfe. Academic, N.Y. (1972). V. 3.

[25] П.А. Марковин, В.А. Трепаков, А.К. Таганцев, А. Дейнека, Д.А. Андреев. ФТТ 58, 131 (2016).

[26] P.A. Markovin, V.A. Trepakov, M.E. Guzhva, A.G. Razdobarin, A.K Tagantsev, D.A. Andreev, A. Dejneka. Mater. Res. Exp. 3, 115705 (2016).

[27] П.А. Марковин, Р.В. Писарев. ЖЭТФ 77, 2461 (1979).

[28] R.V. Pisarev, B.B. Krichevtzov, P.A. Markovin, O.Yu. Korshunov, J.F. Scott. Phys. Rev. B 28, 2677 (1983).

[29] K.A. Muller, W. Berlinger, M. Capizzi, H. Granicher. Solid State Commun. 8, 549 (1970).

[30] U. Bianchi, J. Dec, W. Kleemann, J.G. Bednorz. Phys. Rev. B 51, 8737 (1995).

[31] О.Е. Квятковский. ФТТ 44, 1087 (2002).

[32] О.Ю. Коршунов, П.А. Марковин, Р.В. Писарев. ФТТ 25, 2134 (1983).
[33] O.Yu. Korshunov, P.A. Markovin, R.V. Pisarev. Ferroelectrics Lett. 13, 137 (1992).

[34] O.Yu. Korshunov, P.A. Markovin, R.V. Pisarev, L.M. Sapoznikova. Ferroelectrics 90, 151 (1989).

[35] R.V. Pisarev, P.A. Markovin, B.N. Shermatov, V.I. Voronkova, V.K. Yanovskii. Ferroelectrics 96, 181 (1989).

[36] А.С. Сонин, А.С. Василевская. Электрооптические кристаллы. Атомиздат, М. (1971). 328 с.

[37] П.А. Марковин, М.Е. Гужва. ФТТ 58, 136 (2016).

[38] N. Sai, D. Vanderbilt. Phys. Rev B 62, 13942 (2000).

[39] R.A. Evarestov, E. Blokhin, D. Gryaznov, E.A. Kotomin. J. Maier. Phys. Rev. B 83, 134108 (2011).

[40] http://www.quantum-espresso.org

[41] J.P. Perdew, K. Burke, M. Ernzerhof. Phys. Rev. Lett. 77, 3865 (1996).

[42] H.J. Monkhorst, J.D. Pack. Phys. Rev. B 13, 5188 (1976).

[43] P. Giannozzi, S. Baroni, N. Bonini et al. J. Phys.: Condens. Matter 21, 395502 (2009). http://arxiv.org/abs/0906.2569

[44] Y. Fuji, T. Sakudo. J. Appl. Phys. 41, 4118 (1970).

[45] J.E. Geusiz, S.K. Kuttz, L.G. Van Uitert, S.H. Wemple. Appl. Phys. Lett. 4, 141 (1964).

[46] A. Burgel, W. Kleemann, U. Bianchi. Phys. Rev. B 53, 5222 (1996).

Редактор Т.Н. Василевская 